\title{
Generalized Gibbs Ensemble of 2D CFTs with U(1) charge from the AGT correspondence
}

\author{
Fábio Novaes \\ Centro de Estudios Científicos, \\ Valdivia, Chile \\ E-mail: novaes@cecs.cl
}

Abstract: The Generalized Gibbs Ensemble (GGE) is relevant to understand the thermalization of quantum systems with an infinite set of conserved charges. In this work, we analyze the GGE partition function of 2D Conformal Field Theories (CFTs) with a U(1) charge and quantum Benjamin-Ono $2\left(\mathrm{qBO}_{2}\right)$ hierarchy charges. We use the Alday-GaiottoTachikawa (AGT) correspondence to express the thermal trace in terms of the Alba-FateevLitvinov-Tarnopolskiy (AFLT) basis of descendants, which diagonalizes all charges. We analyze the GGE partition function in the thermodynamic semiclassical limit, including the first order quantum correction. We find that the equality between GGE averages and primary eigenvalues of the $\mathrm{qBO}_{2}$ charges is attainable in the strict large $c$ limit and potentially violated at the subleading $1 / c$ order. We also obtain the finite $c$ partition function when only the first non-trivial charge is turned on, expressed in terms of partial theta functions. Our results should be relevant to the eigenstate thermalization hypothesis for charged CFTs, Warped CFTs and effective field theory descriptions of condensed matter systems.

KEywords: Conformal Field Theory, Integrable Hierarchies, Conformal and W Symmetry

ArXiv EPRINT: 2103.13943 


\section{Contents}

1 Introduction 1

2 The quantum Benjamin-Ono 2 integrable hierarchy 4

2.1 AFLT basis for descendants 5

$2.2 \quad \mathrm{qBO}_{2}$ charges and eigenvalues 8

$\begin{array}{lll}2.2 .1 & \text { Spectrum domains and equilibrium } & 10\end{array}$

3 Generalized Gibbs Ensemble of the $\mathrm{qBO}_{2}$ hierarchy 12

3.1 Thermodynamic limit 14

$\begin{array}{ll}\text { 3.2 GGE free energy in the thermodynamic semiclassical limit } & 16\end{array}$

$\begin{array}{ll}3.3 \text { Leading quantum correction } & 19\end{array}$

$\begin{array}{lll}3.4 & \text { Free energy at finite } c \text { with non-zero } \eta_{2} & 22\end{array}$

4 Generalized eigenstate thermalization for the $\mathrm{qBO}_{2}$ charges in the semiclassical limit $\quad 24$

$\begin{array}{lll}5 & \text { Discussion } & 27\end{array}$

$\begin{array}{ll}\text { A Derivation of integrals of motion and analytic ordering } & 29\end{array}$

A.1 Derivation of $Q_{2}$

A.2 Derivation of $Q_{3} \quad 36$

$\begin{array}{ll}\text { B Combinatorial identities of partitions } & 37\end{array}$

\section{Introduction}

The emergence of thermodynamics from interacting quantum systems is one of the most fundamental and intriguing topics in physics. While open quantum systems can be argued to equilibrate with an external heat bath, isolated quantum systems are harder to adjust to the usual statistical mechanics arguments. The traditional understanding is that quantum integrable systems do not thermalize due to the existence of a infinite number of conserved charges, contrary to chaotic systems. This notion can be put into more concrete terms in a precise hypothesis: if an isolated quantum system obeys the eigenstate thermalization hypothesis (ETH) [1-3], it should dynamically thermalize. The ETH is essentially a statement on the condition for quantum expectation values to approximately match ensemble averages in the thermodynamic limit. More precisely, given a local operator $\mathcal{O}$ and an energy eigenbasis $\left|E_{n}\right\rangle$, the ETH can be mathematically stated as

$$
\left\langle E_{n}|\mathcal{O}| E_{m}\right\rangle=\mathcal{O}_{\mathrm{th}} \delta_{n m}+e^{-S} \delta_{\mathcal{O}}\left(E_{n}, E_{m}\right) R_{n m}
$$


where $\mathcal{O}_{\text {th }}$ is a thermal average, $S$ is the entropy, $\delta_{\mathcal{O}}$ is a slowly varying function of its arguments and $R_{n m}$ is a random matrix of zero mean and unit variance [3]. Strong universal properties are supposed to exist to make this hypothesis true for a large class of quantum systems. In the case the theory has global symmetries, we should consider the charged version of ETH. For neutral operators, the charged ETH is essentially equivalent to the version above with the appropriate dependence on the charges [4].

In the context of $2 \mathrm{D}$ conformal field theories (CFTs), one can argue that minimal models are integrable and do not thermalize [5]. On the other hand, given the AdS/CFT duality, one expects that CFTs with large central charge should thermalize, consistent with black holes behaving as thermal systems. If one restricts the attention to the operators in the CFT vacuum family, detailed investigations showed that the ETH is indeed satisfied in the large c limit of holographic 2D CFTs [6-8]. This match claims that such CFTs are chaotic [9-11], suggesting distinctive criteria for a CFT to have a gravitational dual. The justification for focusing on the vacuum family is that the thermal expectation values for generic operators should vanish in the thermodynamic limit. However, there are indications that the ETH matching for the vacuum family fails for finite central charge in the usual micro and canonical ensembles [6-8]. This opens up the question of what is the proper formulation of ETH for 2D CFTs.

For generic primary operators $\mathrm{O}$, the matrix elements in (1.1) are related to OPE coefficients. Thus, the most general formulation of ETH for 2D CFTs is one about the statistics of OPE coefficients. One can then prove an averaged version of ETH by integrating these coefficients over an order one energy window $[12,13]$. However, the original ETH equality between ensembles has to be proven in an exponentially small energy window, i.e., effectively for a single eigenstate, and this has not been proven in full generality yet (see [14] for a related discussion outside the CFT context). ${ }^{1}$ In this work, we focus on questions of typicality of states in a thermal ensemble with conserved charges, which might be useful to analyze the ETH for the vacuum family, but not necessarily for generic operators.

2D Virasoro CFTs have an infinite set of commuting charges from the quantum Korteweg-de Vries (qKdV) hierarchy, evidence of a rich integrable structure [15-17]. That could be the reason why the ensemble matching mentioned above fails for finite $c$, as a large class of states keep memory of its initial conditions via those integrable charges [18]. The notion of a generalized ETH has been proposed to account for equilibration in quantum integrable systems [19-23]. This formulation compares eigenstate averages with Generalized Gibbs Ensemble (GGE) averages, instead of the canonical ensemble, which is defined as a grand canonical ensemble with an infinite set of charges and associated chemical potentials.

In this work, we will consider the GGE defined in terms of mutually commuting charges $\left\{Q_{k}\right\}_{k \geq 0}$ obtained from symmetry currents, where $Q_{0} \equiv \mathcal{J}$ is a U(1) current, $Q_{1} \equiv H$ is the CFT Hamiltonian and $\left\{Q_{k}\right\}_{k>1}$ form an infinite tower of higher spin conserved charges. We want to compare the averages over some common eigenstate $|E, q\rangle$ of all charges

$$
\langle E, q|\mathcal{O}| E, q\rangle \equiv f_{\mathcal{O}}\left(Q_{k}(E, q)\right)
$$

\footnotetext{
${ }^{1}$ We thank the anonymous referee for these remarks on the ETH for generic operators.
} 
where $E$ is the energy, $q$ is the $\mathrm{U}(1)$ charge and $f_{\mathcal{O}}$ is a smooth function of the charges, with a generalized Gibbs ensemble average

$$
\langle\mathcal{O}\rangle_{\mathrm{GGE}} \equiv \frac{1}{Z(\boldsymbol{\eta})} \operatorname{Tr}\left(\mathcal{O} e^{\sum_{k \geq 0} \eta_{k} Q_{k}}\right),
$$

where $\boldsymbol{\eta}=\left(\eta_{0}, \eta_{1}, \eta_{2}, \ldots\right)$ are generalized chemical potentials, with $\eta_{0} \equiv \eta$ being the $\mathrm{U}(1)$ chemical potential, $\eta_{1} \equiv-\beta$ being the inverse temperature and the partition function given by

$$
Z(\boldsymbol{\eta})=\operatorname{Tr}\left(e^{\sum_{k \geq 0} \eta_{k} Q_{k}}\right) .
$$

In particular, we will focus on the averages of the conserved charges themselves, i.e. $\mathcal{O}=Q_{k}$, such that

$$
\left\langle Q_{k}\right\rangle(\boldsymbol{\eta})=\partial_{\eta_{k}} \log Z(\boldsymbol{\eta}) .
$$

The qKdV GGE and its generalized ETH have been recently investigated in the 2D CFT literature by [5, 18, 24-29]. The overall conclusion is that the generalized ETH also only works at leading order in the large central charge limit, in particular for the vacuum family, i.e., descendants of the identity operator. Therefore, our results should be interesting to analyze these quasi-primary operators, but do not address the stronger statements on the generalized ETH for generic operators, which still remains as an open problem. We should also mention the papers [30,31], which discuss CFT thermalization arguing that heavy descendants are the most typical states in the thermodynamic limit. Here we will consider that the typical eigenstates in the thermodynamic semiclassical limit are primaries.

A crucial challenge in the investigation of generalized ETH for 2D CFTs is having better control of finite $c$ GGE averages. We provide advancements in this direction focusing on 2D CFTs with a U(1) current, henceforth called charged CFTs. A natural integrable hierarchy associated to this case is the quantum Benjamin-Ono $\left(\mathrm{qBO}_{2}\right)$ hierarchy $[32,33]$, whose relevance came to light via a CFT proof [33] of the AGT correspondence [34]. This correspondence states the equivalence of partition functions of $\mathcal{N}=2$ four-dimensional supersymmetric quiver gauge theories and Liouville correlation functions, using Nekrasov's instanton partition functions [35]. The full AGT correspondence reproduces Liouville theory correlators, including its precise structure constants. On the other hand, it also provides an alternative combinatorial expansion for CFT conformal blocks using the Alba-FateevLitvinov-Tarnopolskiy (AFLT) basis of $\operatorname{Vir} \times \mathfrak{u}(1)$ descendants [33], where Vir is the Virasoro algebra. Perhaps surprisingly, the AFLT basis diagonalizes all the $\mathrm{qBO}_{2}$ conserved charges. The complete set of eigenvalues of this hierarchy has been conjectured in [36] as a particular case of the quantum $\mathfrak{g l}(2)$ Intermediate Long Wave $\left(\mathrm{qILW}_{2}\right)$ hierarchy. The $\mathrm{qILW}_{2}$ spectrum was defined in terms of Bethe ansatz equations later proven in [37] (see also [38]). The AFLT basis allow us to obtain exact expressions for matrix elements and the GGE partition function at finite $c$, which is the major technical point of this paper. We notice that the charges obtained in [33] did not include the U(1) zero modes, relevant for our GGE discussion, and so we fill this gap by deriving the first three $\mathrm{qBO}_{2}$ charges with U(1) zero modes in appendix A. To achieve this, we slightly reformulate the algorithm of [39] in terms of OPEs and including the U(1) terms. 
We also discuss the validity of the generalized ETH for the $\mathrm{qBO}_{2}$ charges in the sense of (1.1). This requires analyzing the thermodynamic and large central charge limits of the conserved charges. Using a saddle-point calculation, we reach the conclusion that the generalized ETH for the charges is valid in the strict large central charge limit, similar to the $\mathrm{qKdV}$ results $[24,25]$. In particular, if we discard the $\mathrm{U}(1)$ zero modes, the $\mathrm{qBO}_{2}$ eigenvalues reduce to the $\mathrm{qKdV}$ ones in the thermodynamic semiclassical limit, and thus both ensembles become equivalent (see section 3.2). The sub-leading corrections in the central charge can also be written in terms of free boson representations, similar to the $\mathrm{qKdV}$ case [27]. However, here we see that the finite $c \mathrm{qBO}_{2}$ eigenvalues have more complicated free boson representations, briefly discussed in appendix B, which complicates the full integration of the partition function. We identify a potential violation of the generalized ETH for the first 1/c correction, but we had to rely on a particular ansatz for the saddle-point, leaving this question still open in general. Therefore, this work sets a new route to investigate the generalized ETH for charged CFTs at finite $c$ using the AGT correspondence. Other potential applications of our results are on generalized thermalization of Warped CFTs [40] and holographic realizations of integrable hierarchies [41-45]. The quantum $\mathrm{BO}_{2}$ hierarchy has also been conjectured to describe non-linear dynamics of quantum liquids [32, 46-49] and we hope our results can also be relevant in this context.

Here we review the contents of this paper. In section 2, we review the $\mathrm{qBO}_{2}$ hierarchy, its conserved charges, eigenstates and eigenvalues. We use the Liouville parametrization for the CFT parameters, as done in the AGT correspondence, distinguishing the two unitary branches of the spectrum. We show explicitly the first three $\mathrm{qBO}_{2}$ eigenvalues in terms of the Liouville momentum, the $\mathrm{U}(1)$ charge and the two partitions labelling descendant states. These eigenvalues are generically complex and we discuss conditions to make them real, as expected for equilibrium states. In section 3, we analyze the $\mathrm{qBO}_{2}$ GGE in the thermodynamic limit. We remark that the eigenvalues have different limits depending on the unitary branch of the spectrum. We then obtain the free energy in the large central charge limit and its first $1 / c$ correction from a saddle-point calculation. We obtain explicit expressions assuming that only the first two non-trivial chemical potentials $\eta_{2}$ and $\eta_{3}$ are non-zero. We also obtain an exact finite $c$ partition function when only $\eta_{2}$ is nonzero, written in terms of partial theta functions after integrating over the descendants. In section 4, we discuss the generalized ETH for the $\mathrm{qBO}_{2}$ charges. We reach the conclusion that it is valid in the strict large central limit, but that it potentially fails at the $1 / c$ order. Finally, in section 5, we discuss how our results can be used to study generalized thermalization of 2D charged CFTs, Warped CFTs and effective field theories in condensed matter systems. In appendix A, we review how to derive the $\mathrm{qBO}_{2}$ conserved charges using analytic ordering and in appendix B, we derive some useful combinatorial identities for the analysis of the descendant eigenvalues.

\section{The quantum Benjamin-Ono 2 integrable hierarchy}

The notion of integrability has many forms in quantum field theories (QFTs). In its simplest definition, the integrable structure appears as an infinite set of mutually commuting 
conserved charges built out of a finite number of fundamental fields. The KdV equation is part of an integrable hierarchy, an infinite set of equations of motion generated by the aforementioned set of conserved charges. While the well-known KdV equation describes non-linear waves in a shallow channel, the not so widely known Benjamin-Ono (BO) equation describes internal waves in a deep channel [50, 51]. The Intermediate Long Wave (ILW) equation $[52,53]$ is a generalization of these two equations interpolating between both regimes, with the channel depth as a free parameter. The ILW equation is also part of an integrable hierarchy generalizing the $\mathrm{BO}$ and $\mathrm{KdV}$ ones, which in its turn can be generalized to hierarchies with a multiple number of fundamental fields [54]. A generalization of the BO hierarchy with two fundamental fields has been rediscovered from the analysis of Calogero-Sutherland fluids in [32], henceforth called the $\mathrm{BO}_{2}$ hierarchy, proposed to be relevant for effective low-energy descriptions of condensed matter systems.

All the above mentioned classical integrable hierarchies have a quantum version, which can be built out of Virasoro and current algebras. In the case of 2D CFTs, the natural integrable hierarchy is the qKdV hierarchy [15-17], whose conserved charges are integrals of appropriate polynomials of the energy-momentum tensor $T(z)$ and its derivatives (similarly to its anti-holomorphic counterparts). Likewise, the $\mathrm{qBO}_{2}$ integrable hierarchy is a natural one for 2D CFTs with an extra $\mathrm{U}(1)$ current $J(z)$ [33]. As in the classical case, the qILW 2 hierarchy gives the $\mathrm{qBO}_{2}$ hierarchy in one limit and the commuting $\mathrm{qKdV}$ and free boson hierarchies in the other limit. In this work, we focus on the $\mathrm{qBO}_{2}$ hierarchy as its spectrum has a simpler form than the $\mathrm{qILW}_{2}$ one, both derived in [36]. For other interesting applications of these integrable hierarchies in supersymmetric theories, see, for example, [55-58].

The $\mathrm{qBO}_{2}$ charges were first calculated in [33] and its eigenvalues were obtained in [36]. Let us review this construction in the following, with the particular addition of the U(1) zero modes not considered before.

\subsection{AFLT basis for descendants}

The tensor product algebra $\mathcal{A}=\operatorname{Vir} \otimes \mathfrak{u}(1)$, where Vir is the Virasoro algebra, is generated by the Virasoro and $\mathrm{U}(1)$ currents defined on the cylinder

$$
T(x)=\sum_{n=-\infty}^{\infty} L_{n} e^{-i n x}-\frac{c}{24}, \quad J(x)=\sum_{n=-\infty}^{\infty} a_{n} e^{-i n x}, \quad x \in(0,2 \pi),
$$

with modes obeying the commutation relations

$$
\begin{aligned}
{\left[L_{n}, L_{m}\right] } & =(n-m) L_{n+m}+\frac{c}{12} n\left(n^{2}-1\right) \delta_{n,-m}, \\
{\left[a_{n}, a_{m}\right] } & =\frac{n}{2} \delta_{n,-m}, \quad\left[a_{n}, L_{m}\right]=0 .
\end{aligned}
$$

A primary state is here denoted by $\left|v_{P, a}\right\rangle$ and obeys the relations

$$
\begin{aligned}
a_{0}\left|v_{P, a}\right\rangle & =a\left|v_{P, a}\right\rangle, & a_{n}\left|v_{P, a}\right\rangle & =0, n>0, \\
L_{0}\left|v_{P, a}\right\rangle & =\Delta(P)\left|v_{P, a}\right\rangle, & L_{n}\left|v_{P, a}\right\rangle & =0, n>0
\end{aligned}
$$


where the Liouville parametrization for the central charge $c$ and conformal weights $\Delta$ is given by

$$
c=1+6 Q^{2}, \quad Q=b+\frac{1}{b}, \quad \Delta(P)=\frac{Q^{2}}{4}-P^{2},
$$

with Liouville parameter $b$ labeling the central charge and the momentum $P$ labeling the conformal weights $\Delta(P)$ of primary states. An equivalent definition follows for the anti-holomorphic modes with eigenvalues $\bar{\Delta}(\bar{P})$ and $\bar{a}$. These conventions can be used to describe the spectrum of any CFT if we properly specify $b$ and the domain of $P$ and $a$, which can also be discrete in the case of minimal models (for a recent CFT treatise using this notation, see [59]). We also mention that the Liouville parametrization (2.4) is invariant under $b \rightarrow 1 / b$. Thus, there are two ways to get the semiclassical limit $c \rightarrow \infty$, using either $b \rightarrow 0$ or $b \rightarrow \infty$. In this work, we will only consider the $b \rightarrow 0$ limit.

Assuming that $c>1$, we distinguish two unitary branches of the spectrum with $\Delta>0$

$$
\begin{aligned}
\text { Real branch: } & \mathcal{S}_{R}=\left\{P \in \mathcal{S} \subseteq\left(-\frac{Q}{2}, \frac{Q}{2}\right), Q \in \mathbb{R}\right\}, \\
\text { Liouville branch: } & \mathcal{S}_{L}=\{P=i p, \quad p \in \mathcal{S} \subseteq \mathbb{R}\},
\end{aligned}
$$

where $\mathcal{S}$ is some discrete or continuum subset of $\mathbb{R}$. The AGT formulas are more conveniently written with respect to $P$, but later we will focus on the unitary thermodynamic semiclassical limit, which is only attainable in the Liouville branch $P=i p$. To avoid extra degeneracy in the spectrum, we shall consider $p \geq 0$. With respect to the $\mathrm{U}(1)$ charge $a$, we shall initially assume that it is either real or purely imaginary. As we will see below, the spectrum of the $\mathrm{qBO}_{2}$ charges can be complex and unbounded. The mitigation of these potentially unphysical properties depends on further assumptions on the domains of $p$ and $a$, related to the convergence of the partition function. We do not have a general solution for this, but we will exemplify how it can be solved for the first two non-trivial $\mathrm{qBO}_{2}$ charges in section 2.2.1. We will also discuss convergence properties of the partition function and boundedness of the free energy in section 3 .

The standard basis for the descendant states, henceforth called just descendants, is

$$
a_{-\lambda_{m}} \cdots a_{-\lambda_{1}} L_{-\mu_{n}} \cdots L_{-\mu_{1}}\left|v_{P, a}\right\rangle, \quad \lambda_{1} \geq \lambda_{2} \geq \cdots \geq \lambda_{m}>0, \quad \mu_{1} \geq \mu_{2} \geq \cdots \geq \mu_{n}>0,
$$

where $\lambda=\left(\lambda_{1}, \lambda_{2}, \ldots, \lambda_{m}\right)$ and $\mu=\left(\mu_{1}, \mu_{2}, \ldots, \mu_{n}\right)$ are two independent partitions of an integer. To simplify the notation, we introduce a multi-index $I=(\lambda, \mu)$ such that a descendant at level $|I| \equiv|\lambda|+|\mu|$, with $|\lambda|=\sum_{i} \lambda_{i}$ and $|\mu|=\sum_{i} \mu_{i}$, is defined by

$$
\left|v_{P, a}\right\rangle_{I} \equiv a_{-\lambda_{m}} \cdots a_{-\lambda_{1}} L_{-\mu_{n}} \cdots L_{-\mu_{1}}\left|v_{P, a}\right\rangle .
$$

This basis has a natural Hermitean inner product if we use the prescription

$$
L_{n}^{\dagger}=L_{-n}, \quad a_{n}^{\dagger}=a_{-n},
$$

such that the dual vector of $(2.7)$ is

$$
{ }_{I}\left\langle v_{P, a}\right| \equiv\left\langle v_{P, a}\right| L_{\mu_{n}} \cdots L_{\mu_{1}} a_{\lambda_{m}} \cdots a_{\lambda_{1}} .
$$


The Kac-Shapovalov matrix of inner products is then level-orthogonal

$$
M_{I, J}(\Delta(P))={ }_{I}\left\langle v_{P, a} \mid v_{P, a}\right\rangle_{J} \propto \delta_{|I|,|J|},
$$

where the Kronecker delta denotes its block-diagonal form. As the Virasoro and U(1) algebras commute, the inner product decomposes into two independent inner products. The $\mathrm{U}(1)$ inner product is positive definite in our conventions and the $\mathrm{U}(1)$ zero mode plays no role in this case. The Virasoro inner product is also positive definite for $c>1$ if $\Delta>0[60,61]$. Therefore, there are no null or negative states if $c>1, \Delta>0$ and $a$ arbitrary, implying that the CFT spectrum is unitary.

The usual convention in the charged CFT literature is to define the Virasoro modes in terms of a total energy-momentum tensor $T^{\prime}=T+\frac{J^{2}}{\kappa}$, with $\kappa$ being the Kac-Moody level. In this case, the Virasoro modes do not commute with the U(1) modes

$$
\begin{aligned}
{\left[L_{n}^{\prime}, L_{m}^{\prime}\right] } & =(n-m) L_{n+m}^{\prime}+\frac{c+1}{12} n\left(n^{2}-1\right) \delta_{n,-m}, \\
{\left[a_{n}, a_{m}\right] } & =\kappa \frac{n}{2} \delta_{n,-m}, \quad\left[a_{n}, L_{m}^{\prime}\right]=n a_{n+m},
\end{aligned}
$$

and the spectrum unitarity depends on the domains of both $P$ and $a$, which is particularly relevant to the study of Warped CFTs [40, 62, 63]. However, as we discuss in section 3 , the partition function is invariant under this change of basis and our results are also valid for (2.11). Therefore, we will mostly focus on the commuting algebra (2.2), equivalent to (2.11) with $\kappa=1$ up to this change of basis, which greatly simplify the computations.

Now, we introduce the AFLT basis [33] as special linear combinations of the descendants $(2.7)$

$$
\left|w_{P, a}\right\rangle_{I}=\sum_{J \in \mathbb{Y}_{|I|}} C_{I}^{J}(P)\left|v_{P, a}\right\rangle_{J}
$$

where $\mathbb{Y}_{|I|}$ is the set of all partitions of size $|I|$. The first few coefficients $C_{I}^{J}(P)$ are given in [33] and can be derived from the recursive algorithm described there. The Hermitean conjugate of (2.12) depends on the proper domain of $P$. However, in the AFLT construction, the dual basis is defined without complex conjugation of the coefficients as

$$
{ }_{I}\left\langle w_{P, a}^{*}\right|=\sum_{J \in \mathbb{Y}_{|I|}}\left\langle v_{P, a}\right| C_{I}^{J}(P),
$$

which we distinguish from the usual Hilbert space dual basis ${ }_{I}\left\langle w_{P, a}\right|$ by a star in the vector.

Given a primary field $V_{\alpha}$, the crucial fact of the AFLT basis is that

$$
\frac{{ }_{I}\left\langle w_{P^{\prime}, a}\left|V_{\alpha}\right| w_{P, a}\right\rangle_{J}}{\left\langle w_{P^{\prime}, a}\left|V_{\alpha}\right| w_{P, a}\right\rangle}=Z_{\mathrm{bif}}\left(\alpha \mid P^{\prime}, I ; P, J\right)
$$

where $Z_{\text {bif }}$ is the bifundamental part of the Nekrasov partition function [35] defined as

$$
Z_{\mathrm{bif}}\left(\alpha \mid P^{\prime}, I ; P, J\right)=\prod_{i, j=1}^{2} \prod_{s \in \mu_{i}}\left[Q-E_{\mu_{i}, \lambda_{j}}\left(\sigma_{i} P-\sigma_{j} P^{\prime} \mid s\right)-\alpha\right] \prod_{t \in \lambda_{j}}\left[E_{\lambda_{j}, \mu_{i}}\left(\sigma_{j} P^{\prime}-\sigma_{i} P \mid t\right)-\alpha\right]
$$


where $I=\left(\lambda_{1}, \lambda_{2}\right), J=\left(\mu_{1}, \mu_{2}\right),\left(\sigma_{1}, \sigma_{2}\right)=(1,-1)$ and

$$
E_{\lambda, \mu}(P \mid s)=P-b l_{\mu}(s)+b^{-1}\left(a_{\lambda}(s)+1\right) .
$$

For a given a box $s=(i, j), a_{\lambda}(s)=\lambda_{i}-j$ refers to the arm length of the box $s$ in the partition $\lambda$ and $l_{\mu}(i, j)=\mu_{j}^{\prime}-i$ is the leg length of the box $s$ in the partition $\mu$, where $\mu^{\prime}$ is the transpose partition of $\mu$. One can also show that [33]

$$
{ }_{I}\left\langle w_{P^{\prime}, a}^{*} \mid w_{P, a}\right\rangle_{J}=\mathcal{N}_{I}(P) \delta_{P^{\prime}, P} \delta_{I, J}, \quad \mathcal{N}_{I}(P) \equiv Z_{\mathrm{bif}}(0 \mid P, I ; P, I)
$$

which means that the AFLT basis is orthogonal in this inner product. As a consequence of $(2.12),(2.13)$ and $(2.17)$, we have

$$
\sum_{K, L} C_{I}^{K}(P) M_{K, L}(\Delta(P)) C_{J}^{L}(P)=\mathcal{N}_{I}(P) \delta_{I, J},
$$

or in matrix form

$$
C \cdot M \cdot C=\mathcal{N}
$$

where $\mathcal{N}$ is diagonal, making explicit that the transformation (2.12) diagonalizes the inner product matrix, although not in the usual Hermitean conjugate dual basis.

Taking the determinant of (2.19), we notice that $\mathcal{N}=0$ only for degenerate states [33] and those are out of the unitary spectrum for $c>1$. Therefore, we can redefine ${ }_{I}\left\langle w_{P, a}^{*}\right| \rightarrow$ $\sqrt{\mathcal{N}_{I}(P)}{ }_{I}\left\langle w_{P, a}^{*}\right|$ and make the basis orthonormal. The AFLT states with $I=\left(\lambda_{1}, \varnothing\right)$ and $I=\left(\varnothing, \lambda_{2}\right)$ are equivalent to the Jack basis, eigenfunctions of the Calogero-Sutherland (CS) Hamiltonians [64]. The complete basis has an interpretation in terms of eigenstates of two interacting CS hamiltonians, obtained using a bosonic representation of the Virasoro generators [65].

\section{$2.2 \quad \mathrm{qBO}_{2}$ charges and eigenvalues}

The AFLT states (2.12) are eigenstates of the $\mathrm{qBO}_{2}$ Hamiltonians $Q_{k}$ [33]

$$
Q_{k}=\frac{1}{2 \pi} \int_{0}^{2 \pi} d x \mathcal{Q}_{k+1}(x), \quad k=0,1,2, \ldots,
$$

obtained by integrating the currents $\mathcal{Q}_{k}(x)$, with the first four ones given by

$$
\begin{aligned}
& \mathcal{Q}_{1}=J, \\
& \mathcal{Q}_{2}=T+J^{2}, \\
& \mathcal{Q}_{3}=T J+i Q J \mathcal{H} J_{x}+\frac{1}{3} J^{3}, \\
& \mathcal{Q}_{4}=T^{2}+6 T J^{2}+6 i Q T \mathcal{H} J_{x}+6 i Q J^{2} \mathcal{H} J_{x}-6 Q^{2}\left(\mathcal{H} J_{x}\right)^{2}+\left(1+Q^{2}\right) J_{x}^{2}+J^{4},
\end{aligned}
$$

where $J_{x} \equiv \frac{d J}{d x}$ and $\mathcal{H}$ represents the Hilbert transform on the circle

$$
\mathcal{H} f(x)=\frac{1}{2 \pi} \mathcal{P} \int_{0}^{2 \pi} d y f(y) \cot \frac{1}{2}(y-x),
$$


with $\mathcal{P}$ denoting the Cauchy principal value integral. We regularize currents via analytic ordering $[15,39]$, as described in appendix A. The first three non-trivial charges are given by

$$
\begin{aligned}
Q_{1}= & L_{0}+2 \sum_{k=1}^{\infty} a_{-k} a_{k}+a_{0}^{2}-\frac{c+1}{24}, \\
Q_{2}= & \sum_{n \neq 0} L_{-n} a_{n}+2 i Q \sum_{n>0}|n| a_{-n} a_{n}+\frac{1}{3} \sum_{n+m+p=0}: a_{n} a_{m} a_{p}:+a_{0}\left(L_{0}-\frac{c+1}{24}\right), \\
Q_{3}= & 2 \sum_{n>0} L_{-n} L_{n}+L_{0}^{2}-\frac{c+5}{12} L_{0}+\frac{5 c^{2}+42 c+37}{2880}+6 \sum_{m \neq 0} \sum_{n+p=m} L_{-m} a_{n} a_{p}+ \\
& +6\left(L_{0}-\frac{c+1}{24}\right)\left(2 \sum_{n>0} a_{-n} a_{n}+a_{0}^{2}\right)+6 i Q \sum_{n \neq 0}|n| L_{n} a_{-n}+\sum_{n>0}: \sum_{m+n}: a_{m} a_{n} a_{p} a_{q}: .
\end{aligned}
$$

The eigenvalues of the charges (2.23) have the generic form

$$
E_{I}^{(k)}(P, a)=E^{(k)}(P, a)+e_{I}^{(k)}(P, a) \quad(k \geq 0),
$$

where we call $E^{(k)}(P, a)$ the primary part, polynomials in $\Delta(P)$ and $a$, while $e_{I}^{(k)}(P, a)$ is the descendant part, being zero for primary states. The first four eigenvalues with $a=0$ were presented explicitly in [36] and here we extend that result to non-zero $a$. The descendant part can be written in terms of the charged Calogero-Sutherland energies defined as

$$
h_{(\lambda, \mu)}^{(k)}(P, a)=h_{\lambda}^{(k)}(P, a)+h_{\mu}^{(k)}(-P, a)
$$

with

$$
h_{\lambda}^{(k)}(P, a)=b^{1-k} \sum_{j>0}\left\{\left[b(P-i a)-\frac{b^{2}}{2}+\lambda_{j}+j b^{2}\right]^{k}-\left[b(P-i a)-\frac{b^{2}}{2}+j b^{2}\right]^{k}\right\} .
$$

The first eigenvalue is trivial $E_{I}^{(0)}(P, a)=E^{(0)}(a)=a$, while the first three non-trivial eigenvalues with nonzero $a$ are

$$
\begin{aligned}
& E_{(\lambda, \mu)}^{(1)}(P, a)=E^{(1)}(P, a)+h_{(\lambda, \mu)}^{(1)}(P, a), \\
& E_{(\lambda, \mu)}^{(2)}(P, a)=E^{(2)}(P, a)+\frac{i}{2} h_{(\lambda, \mu)}^{(2)}(P, a), \\
& E_{(\lambda, \mu)}^{(3)}(P, a)=E^{(3)}(P, a)-2 h_{(\lambda, \mu)}^{(3)}(P, a)-\frac{1+b^{2}}{2} h_{(\lambda, \mu)}^{(1)}(P, a),
\end{aligned}
$$


where

$$
\begin{aligned}
& E^{(1)}(P, a)=\Delta(P)+a^{2}-\frac{c+1}{24}, \\
& E^{(2)}(P, a)=a\left(\Delta(P)-\frac{c+1}{24}\right)+\frac{1}{3} a^{3}, \\
& E^{(3)}(P, a)=\Delta(P)^{2}-\frac{c+5}{12} \Delta(P)+\frac{5 c^{2}+42 c+37}{2880}+6 a^{2}\left(\Delta(P)-\frac{c+1}{24}\right)+a^{4} .
\end{aligned}
$$

The above eigenvalues were verified using computer algebra.

Given the known results for the $a=0$ eigenvalues in the literature [33, 36, 37], we expect that the descendant part for $k>3$ can be written as a linear combination of the $h_{(\lambda, \mu)}^{(q)}(P, a)$ with $q \leq k$, although the explicit coefficients are not known in general. This should come as a consequence of the eigenvalues being symmetric polynomials in the Bethe roots described in $[36,37]$. An informal argument for this structure comes from the fact that the $\mathrm{qBO}_{2}$ charges can be written in terms of CS Hamiltonians [65]. Moreover, for the fixed $k$ eigenvalue, the highest partition contribution $\sum_{i} \lambda_{i}^{k}$ comes from the highest power in the current $J^{k+1}$, which sets that only the $h_{(\lambda, \mu)}^{(q)}(P, a)$ with $q \leq k$ contribute.

\subsubsection{Spectrum domains and equilibrium}

The $\mathrm{qBO}_{2}$ energies (2.27) are generically complex, depending on the domain of $P$ and a. Complex eigenvalues in principle correspond to some damping or forcing factor in the Hamiltonian time evolution and thus can model non-equilibrium phenomena in open quantum systems. Therefore, we do not expect imaginary contributions in equilibrium ensembles of an isolated quantum system. This is a physical caveat to the study of the $\mathrm{qBO}_{2}$ GGE ensemble for chiral CFTs like Warped CFTs. However, complex $\mathrm{qBO}_{2}$ eigenvalues can make sense at equilibrium for non-chiral CFTs if the sum of holomorphic and antiholomorphic charges cancels their imaginary parts. That will be our working hypothesis in the rest of this paper. In fact, in section 3 we will compute the semiclassical free energy and cancel its imaginary part a posteriori, as a result of this assumption. We will then check under what conditions the free energy is bounded from below. In the following, we shall analyze if and under what conditions the $\mathrm{qBO}_{2}$ charges $Q_{2}$ and $Q_{3}$ can be made real and bounded and discuss implications for chiral CFTs.

To begin with, it is reasonable to assume that $P$ and $a$ are either real or purely imaginary, as the CFT energy $E_{I}^{(1)}(P, a)$ is real in any of those cases. The Hermiticity condition (2.8) is compatible with $a$ real, but we could also consider $a$ purely imaginary by assuming $a_{n}^{\dagger}=-a_{-n}$. This can generate a unitary spectrum if we restore the Kac-Moody parameter $\kappa$ and let $\kappa \rightarrow-\kappa$, as discussed, for example, in the Warped CFT case [63]. Therefore, let us consider the four possibilities for the pair $(P, a)$, in which they are either real or imaginary separately, and see what this implies for the higher $\mathrm{qBO}_{2}$ eigenvalues.

The simplest case to consider is that both $P$ and $a$ are real. Then, the imaginary parts of the eigenvalues come from terms with an odd number of Hilbert transforms in the $\mathrm{qBO}_{2}$ charges (2.23) and the imaginary coefficients in the AFLT basis (2.12) (see [33] below their 
eq. (2.14) for explicit expressions). These result in imaginary parts in (2.27), which cannot be cancelled in chiral CFTs.

If $P$ is imaginary and $a$ is either real or imaginary, the conclusions are the same and we still have imaginary parts for generic partitions. However, if $P$ is real and $a$ is purely imaginary, the Calogero energies (2.26) are real, as the AFLT coefficients in (2.12) can all be made real. On the other hand, the primary parts of the even $k$ charges have only odd powers of $a$ and are thus purely imaginary. As we can see from (2.27), the $k=2$ descendant part is also purely imaginary. This charge can thus be made real if we let $\eta_{2} \rightarrow i \eta_{2}$ (this was effectively done in [43]). This pattern seems to be present for any even $k$ and, therefore, the case $P$ real and $a$ imaginary apparently is the only one in which all $\mathrm{qBO}_{2}$ charges can be made real by properly choosing the chemical potentials. This thus seems to be the ideal case for the $\mathrm{qBO}_{2}$ equilibrium ensemble in chiral CFTs. Although $P$ real is incompatible with a unitary thermodynamic limit in which $P \gg \ell$, with $\ell$ the size of the cylinder, as this would generate negative values of $\Delta$, it could make sense if $P$ only scales with the central charge and not with $\ell$. It would be interesting to further investigate this possibility for chiral CFTs in the future.

Clearly, we can still get real total charges in any domain if we sum the holomorphic charges with their anti-holomorphic counterparts. Let us exemplify this by first analyzing the imaginary part of $E_{(\lambda, \mu)}^{(2)}$ in (2.27). Expanding (2.26) for $k=2$, we get

$$
\begin{aligned}
h_{\lambda}^{(2)}(P, a) & =b^{-1} \sum_{j} \lambda_{j}^{2}+2(P-i a)|\lambda|+b \sum_{j}(2 j-1) \lambda_{j} \\
& =b^{-1} \sum_{j} \lambda_{j}^{2}+2(P-i a)|\lambda|+b \sum_{j} \lambda_{j}^{\prime 2},
\end{aligned}
$$

where we used the identity (see appendix B)

$$
\sum_{j}(2 j-1) \lambda_{j}=\sum_{j} \lambda_{j}^{\prime 2}
$$

with $\lambda^{\prime}$ being the conjugate partition of $\lambda$. Then, from (2.25) and (2.27), we have

$$
\begin{aligned}
\operatorname{Im} E_{(\lambda, \mu)}^{(2)}= & \operatorname{Im} E^{(2)}+ \\
& +\frac{1}{2}\left[b^{-1} \sum_{j}\left(\lambda_{j}^{2}+\mu_{j}^{2}\right)+2(\operatorname{Im} a+\operatorname{Re} P)|\lambda|+2(\operatorname{Im} a-\operatorname{Re} P)|\mu|+b \sum_{j}\left(\lambda_{j}^{\prime 2}+\mu_{j}^{\prime 2}\right)\right] .
\end{aligned}
$$

If the descendant contribution is diagonal between the sectors, the sum of both sectors cancels the imaginary part in the GGE partition function. More explicitly, if $\eta_{2}$ is real, the total real eigenvalue is

$$
\eta_{2}\left(E_{(\lambda, \mu)}^{(2)}+\bar{E}_{(\lambda, \mu)}^{(2)}\right)=2 \eta_{2}\left[\operatorname{Re} E^{(2)}+(\operatorname{Re} a-\operatorname{Im} P)|\lambda|+(\operatorname{Re} a+\operatorname{Im} P)|\mu|\right],
$$

and if $\eta_{2} \rightarrow i \eta_{2}$, the real eigenvalue is actually (2.31). Meanwhile, the charge $Q_{3}$ has a real primary part $E^{(3)}(P, a)$ in any case and the potential imaginary part comes from the $k=3$ 
charged Calogero energy

$$
\begin{aligned}
h_{(\lambda, \mu)}^{(3)}(P, a)= & b^{-2} \sum_{j}\left(\lambda_{j}^{3}+\mu_{j}^{3}\right)+3 P\left(b^{-1} \sum_{j}\left(\lambda_{j}^{2}-\mu_{j}^{2}\right)+b \sum_{j}\left(\lambda_{j}^{\prime 2}-\mu_{j}^{\prime 2}\right)\right)- \\
& -3 i a\left(b^{-1} \sum_{j}\left(\lambda_{j}^{2}+\mu_{j}^{2}\right)+b \sum_{j}\left(\lambda_{j}^{\prime 2}+\mu_{j}^{\prime 2}\right)\right)+3\left(P^{2}-a^{2}-\frac{b^{2}}{12}\right)(|\lambda|+|\mu|)- \\
& -6 i P a(|\lambda|-|\mu|)+\frac{3}{2} \sum_{j}(2 j-1)\left(\lambda_{j}^{2}+\mu_{j}^{2}\right)+b^{2} \sum_{j}\left(\lambda_{j}^{\prime 3}+\mu_{j}^{\prime 3}\right),
\end{aligned}
$$

where we used (2.30) and (see appendix B)

$$
\sum_{j}(2 j-1)^{2} \lambda_{j}=\frac{4}{3} \sum_{j} \lambda_{j}^{\prime 3}-\frac{1}{3}|\lambda| .
$$

If we add the extra descendant part of $Q_{3}$ in (2.27), there is a small cancellation of the term $-\frac{b^{2}}{4}(|\lambda|+|\mu|)$ above and we finally get for the total descendant part

$$
\begin{aligned}
e_{(\lambda, \mu)}^{(3)}(P, a)= & -2 b^{-2} \sum_{j}\left(\lambda_{j}^{3}+\mu_{j}^{3}\right)-6 P\left(b^{-1} \sum_{j}\left(\lambda_{j}^{2}-\mu_{j}^{2}\right)+b \sum_{j}\left(\lambda_{j}^{\prime 2}-\mu_{j}^{\prime 2}\right)\right)+ \\
& 6 i a\left(b^{-1} \sum_{j}\left(\lambda_{j}^{2}+\mu_{j}^{2}\right)+b \sum_{j}\left(\lambda_{j}^{\prime 2}+\mu_{j}^{\prime 2}\right)\right)-6\left(P^{2}-a^{2}+\frac{1}{12}\right)(|\lambda|+|\mu|)+ \\
& +12 i P a(|\lambda|-|\mu|)-3 \sum_{j}(2 j-1)\left(\lambda_{j}^{2}+\mu_{j}^{2}\right)-2 b^{2} \sum_{j}\left(\lambda_{j}^{\prime 3}+\mu_{j}^{\prime 3}\right) .
\end{aligned}
$$

We can get a real eigenvalue by summing both sectors as in the previous case.

Even if we solve the reality problem, there is still a potential stability problem for the equilibrium state for both eigenvalues above, as the real and imaginary parts are not necessarily bounded from below. This could be solved by adding the other $\mathrm{qBO}_{2}$ charges or imposing restrictions on the spectrum, but it is still an open problem in general. This is similar to what happens in the grand canonical ensemble of a free gas, the partition function is only convergent for certain values of the energy and chemical potentials, but here we have a problem of increasingly complexity as we add more charges. In the next section, we will make a semiclassical saddle-point analysis and then find the free energy boundedness conditions a posteriori.

\section{Generalized Gibbs Ensemble of the $\mathrm{qBO}_{2}$ hierarchy}

In this section, we derive the GGE partition function of the $\mathrm{qBO}_{2}$ hierarchy and the free energy in the thermodynamic semiclassical limit. We also obtain a simplified form of the finite $c$ partition function when only $\eta_{2}$ is non-zero. First, let us review the usual CFT construction. The grand canonical partition function of a 2D Euclidean CFT on a cylinder of size $2 \pi \ell$, inverse temperature $\beta$, angular potential $\theta$ and $\mathrm{U}(1)$ charge potential $\eta$ can be 
written as (see [66], for example)

$$
Z(\tau, \eta)=\operatorname{Tr}\left(e^{-\beta H+i \theta \mathcal{M}} e^{i \eta \mathcal{J}}\right)=\operatorname{Tr}\left(q^{L_{0}^{\prime}-\frac{c^{\prime}}{24}} \bar{q}^{\prime}-\frac{c^{\prime}}{24} e^{i \eta \mathcal{J}}\right)
$$

where $q=e^{2 \pi i \tau}$, with the torus moduli parameter defined as $\tau=\frac{1}{2 \pi \ell}(\theta+i \beta)$, and the total Hamiltonian $H$, angular momentum $\mathcal{M}$ and $\mathrm{U}(1)$ charge $\mathcal{J}$ given by

$$
H=\frac{1}{\ell}\left(L_{0}^{\prime}+\bar{L}_{0}^{\prime}-\frac{c^{\prime}}{12}\right), \quad \mathcal{M}=\frac{1}{\ell}\left(L_{0}^{\prime}-\bar{L}_{0}^{\prime}\right), \quad \mathcal{J}=a_{0}-\bar{a}_{0}
$$

and

$$
L_{0}^{\prime} \equiv L_{0}+a_{0}^{2}, \quad c^{\prime}=c+1
$$

This last definition is frequently used in the charged CFT literature, which uses the Virasoro modes of the combined energy-momentum tensor $T^{\prime}=T+\frac{1}{\kappa} J^{2}$ (the Kac-Moody level is $\kappa=1$ in our conventions). In the following, we will discuss only one chiral sector of the partition function and assume the same behaviour for the other sector, which is left implicit in our equations.

The descendant basis (2.7) is neither orthogonal nor normalized, but we must define the partition function as a normalized trace to reflect only the states' degeneracy in its sum. To achieve this, the explicit form of the trace in (3.1) needs to include the inverse of the Kac matrix (2.10) as follows

$$
\begin{aligned}
Z(\tau, \eta) & =\sum_{P, a, I}\left(M^{-1}\right)^{I, I}{ }_{I}\left\langle v_{P, a}\left|e^{2 \pi i \tau\left(L_{0}^{\prime}-\frac{c^{\prime}}{24}\right)+i \eta a_{0}}\right| v_{P, a}\right\rangle_{I} \\
& =\sum_{P, a} e^{2 \pi i \tau\left(\Delta^{\prime}-\frac{c^{\prime}}{24}\right)+i \eta a} \sum_{N=|I|} e^{2 \pi i \tau N} \sum_{I \in \mathbb{Y}_{N}}\left(M^{-1}\right)^{I, I} M_{I, I} \\
& =\sum_{P, a, N} p_{2}^{\prime}(N) e^{2 \pi i \tau\left(\Delta^{\prime}-\frac{c^{\prime}}{24}+N\right)+i \eta a},
\end{aligned}
$$

where the primary eigenvalue of $L_{0}^{\prime}$ is

$$
\Delta^{\prime}(P, a) \equiv \Delta(P)+a^{2}
$$

and $p_{2}^{\prime}(N)$ is the number of partitions of an integer $N$ split into two sub-partitions. The prime in $p_{2}^{\prime}(N)$ means we must exclude the trivial $L_{-1}^{N}$ descendants of the vacuum state $P= \pm Q / 2[67,68]$.

Now let us find the GGE partition function (1.4) by including all the $\mathrm{qBO}_{2}$ charges. As noticed in section 2, these charges are diagonal in the AFLT basis. Let us explicitly show how to write the GGE partition function in the AFLT basis by starting from the 
standard descendant basis

$$
\begin{aligned}
Z(\boldsymbol{\eta}) & =\sum_{P, a, I}\left(M^{-1}\right)^{I, I}{ }_{I}\left\langle v_{P, a}\left|e^{\sum_{k} \eta_{k} Q_{k}}\right| v_{P, a}\right\rangle_{I} \\
& =\sum_{P, a, I} \sum_{J, K}\left(C^{-1}\right)_{I}^{J}\left(M^{-1}\right)^{I, I}\left(C^{-1}\right)_{I}^{K}{ }_{J}\left\langle w_{P, a}^{*}\left|e^{\sum_{k} \eta_{k} Q_{k}}\right| w_{P, a}\right\rangle_{K} \\
& =\sum_{P, a, J}\left(\mathcal{N}_{J}\right)^{-1} \mathcal{N}_{J} e^{\sum_{k} \eta_{k} E_{J}^{(k)}(P, a)} \\
& =\sum_{P, a, J} e^{\sum_{k} \eta_{k} E_{J}^{(k)}(P, a)}
\end{aligned}
$$

where we used (2.12) and (2.13) in the second line and (2.17) and the inverse of (2.19) in the third line. Clearly, we would get the same result if we calculated the trace using the normalized AFLT basis from the beginning.

A similar argument can be used to show that the GGE partition function is the same if we use either the commuting basis in terms of $T$ and $J$ modes or the composite basis in terms of $T^{\prime}=T+J^{2}$ and $J$ modes. It is not difficult to convince oneself that the descendants in terms of $L^{\prime}$ and $a$ operators can be written as linear combinations of the $L$ and $a$ descendants, i.e.,

$$
\left|v_{P^{\prime}, a}^{\prime}\right\rangle_{I}=\sum_{J \in \mathbb{Y}_{|I|}} R_{I, J}\left|v_{P, a}\right\rangle_{J}, \quad{ }_{I}\left\langle v_{P^{\prime}, a}^{\prime}\right|=\sum_{J \in \mathbb{Y}_{|I|}}\left\langle v_{P, a}\right|\left(R^{T}\right)_{I, J}
$$

such that the inner product matrices in the two basis are related by

$$
M^{\prime}\left(\Delta^{\prime}\right)=R^{T} \cdot M(\Delta) \cdot R
$$

Using these results and taking the inverse of (3.8), we can show that

$$
\sum_{P^{\prime}, a, I}\left(M^{\prime-1}\right)_{I, I I}\left\langle v_{P^{\prime}, a}^{\prime}\left|e^{\sum_{k} \eta_{k} Q_{k}}\right| v_{P^{\prime}, a}^{\prime}\right\rangle_{I}=\sum_{P, a, I}\left(M^{-1}\right)_{I, I}{ }_{I}\left\langle v_{P, a}\left|e^{\sum_{k} \eta_{k} Q_{k}}\right| v_{P, a}\right\rangle_{I},
$$

which establishes that the normalized partition function does not depend on such change of basis.

\subsection{Thermodynamic limit}

In this section, we analyze the partition functions above in the thermodynamic limit $\ell \rightarrow \infty$ of the spectrum defined by

$$
\Delta \sim \ell^{2}, \quad N \sim \ell^{2}, \quad a \sim \ell,
$$

where $N \equiv|I|$ refers to the descendant level. The limit (3.10) is consistent with a saddlepoint computation of the CFT free energy in the large $\ell$ limit [24], using the charged Cardy formula $[66,69,70]$ for the asymptotic density of states $\rho\left(\Delta^{\prime}, a\right)$

$$
\log \rho\left(\Delta^{\prime}, a\right) \sim 2 \pi \sqrt{\frac{c^{\prime}}{6}\left(\Delta^{\prime}-\frac{c^{\prime}}{24}-a^{2}\right)}=2 \pi \sqrt{\frac{c+1}{6}\left(\Delta-\frac{c+1}{24}\right)} \sim 2 \pi \sqrt{\frac{c+1}{6} \Delta}
$$


and the asymptotic number of 2-colored partitions [71, 72]

$$
\log p_{2}(N) \sim 2 \pi \sqrt{\frac{N}{3}} .
$$

The charged Cardy formula can be derived assuming that the partition function transforms covariantly under a modular transformation $[66,73]$

$$
Z\left(\frac{A \tau+B}{C \tau+D}, \frac{\eta}{C \tau+D}\right)=e^{\frac{\pi i}{8 \pi^{2}} \frac{C \eta^{2}}{C \tau+D}} Z(\tau, \eta)
$$

The Cardy formula has been rigorously derived recently in $[70,74,75]$, both in the charged and uncharged cases. These works remark that the Cardy asymptotics (3.11) does not distinguish between primaries and descendants. In addition, to get the asymptotics of nonvacuum primaries, we should replace $c$ by $c-1$ in (3.11) [70,74]. We shall use this below. Moreover, [75] shows that the subleading corrections to (3.11) can be sensitive on how the Cardy limit is taken for non-zero spin operators. In this paper, we will only consider the leading order in the thermodynamic limit, so that these subleading terms will not be relevant to our analysis. Therefore, we will effectively assume $\theta=0$ in (3.1).

To derive the free energy $F_{0}$ in the thermodynamic limit (3.10), we can replace the partition function sums in (3.4) by integrals

$$
Z(\tau, \eta) \sim \int d a d \Delta d N e^{2 \pi \sqrt{\frac{c}{6} \Delta}} e^{2 \pi \sqrt{\frac{N}{3}}} e^{-\frac{\beta}{\ell}\left(\Delta+N+a^{2}\right)+i \eta a}=e^{F_{0}},
$$

where we replaced the sum over $P$ by an integral over $\Delta$, which is simpler in this case. In the saddle-point approximation for large $\Delta, a$ and $N$, we get the saddle-point values

$$
\Delta_{*}=\frac{c \pi^{2} \ell^{2}}{6 \beta^{2}}, \quad a_{*}=\frac{i \eta \ell}{2 \beta}, \quad N_{*}=\frac{\pi^{2} \ell^{2}}{3 \beta^{2}}
$$

and the free energy is then

$$
F_{0}=\frac{(c+2) \pi^{2} \ell}{6 \beta}-\frac{\eta^{2} \ell}{4 \beta}
$$

We recover the usual Virasoro CFT result by making $\eta=0$ and $c \rightarrow c-2$, whose origin comes from the shifted Virasoro vacuum energy with $c^{\prime}=c+1$ and the density of descendants which also includes the $\mathrm{U}(1)$ descendants. Notice that, if $\eta$ is real, $a_{*}$ is imaginary in (3.15). This is not a problem per se, but $F_{0}$ is then not bounded from below. Therefore, we will consider that $\eta$ is purely imaginary for the rest of this work.

Let us now consider the thermodynamic limit of the $\mathrm{qBO}_{2}$ charges. As we expressed the GGE partition function (3.6) as a sum over $P$, we should consider the thermodynamic scaling of $P$. In the Liouville branch, $P=i p$ and we will assume that

$$
p \sim \frac{\ell}{b^{2}}, \quad a \sim \frac{\ell}{b^{2}}
$$

such that $\Delta \gg \frac{c-1}{24}$ in the thermodynamic limit. This is consistent with (3.10) and we also introduced the Liouville parameter dependence to be considered in the next section. An 
alternative option would be $p \sim \ell \sqrt{(c-1) / 6} \sim \ell Q \approx \ell / b^{2}$, but, as the $\mathrm{qBO}_{2}$ descendant eigenvalues (2.26) are explicitly written in terms of $b$, it is much more convenient to consider the semiclassical expansion $b \rightarrow 0$ using (3.17).

The scaling (3.17) implies that the primary parts scale as $E^{(k)}(P, a) \sim \ell^{k+1}$ in the thermodynamic limit and lower order terms in (2.28) can be discarded. With respect to the descendant parts $e_{(\lambda, \mu)}^{(k)}$, following a similar argument as in [24], we consider the following effective scaling for the partitions

$$
\sum_{j} \lambda_{j}, \sum_{j} \lambda_{j}^{\prime} \sim \ell^{2}
$$

such that, for example,

$$
\sum_{j} \lambda_{j}^{k} \sim|\lambda|^{1 / 2} \times|\lambda|^{k / 2}=|\lambda|^{(k+1) / 2} \sim \ell^{k+1},
$$

and

$$
\sum_{j}(2 j-1)^{k} \lambda_{j} \sim \sum_{j}{\lambda_{j}^{\prime k+1}}^{k+\ell^{k+2}}
$$

This means that all terms in the Calogero energies (2.26) scale as $h_{(\lambda, \mu)}^{(k)} \sim \ell^{k+1}$, just like the primary parts, and the descendant part $e_{(\lambda, \mu)}^{(k)}$ is thus dominated by $h_{(\lambda, \mu)}^{(k)}$. The $\mathrm{qBO}_{2}$ eigenvalues have thus the following structure in the thermodynamic limit

$$
E_{I}^{(k)} \sim \sum_{q=0}^{\left\lfloor\frac{k+1}{2}\right\rfloor} f_{q}^{(k)} a^{k+1-2 q} \Delta^{q}+d^{(k)} h_{I}^{(k)}(P, a),
$$

with $f_{q}^{(k)}, d^{(k)}$ being constants. Ideally, we would like to integrate the descendant parts in the partition function and then do a saddle-point calculation of the resulting primary character. Finding the coefficients $f_{q}^{(q)}$ and $d^{(k)}$ requires knowing the coefficients of the $\mathrm{qBO}_{2}$ eigenvalues for any $k$, which we do not currently know. Apart from this, the complete integration of the partition function might be done in certain special cases, like when only a finite number of chemical potentials is non-zero. Below we are going to discuss the case when only $\eta_{2}$ and $\eta_{3}$ are non-zero.

\subsection{GGE free energy in the thermodynamic semiclassical limit}

Here we calculate the free energy of the $\mathrm{qBO}_{2} \mathrm{GGE}$ in the semiclassical limit. For a generic non-relativistic critical system, the leading saddle-point could be different from the CFT one, but we will not consider this possibility. We shall proceed by considering the extra $\mathrm{qBO}_{2}$ charges as corrections to the CFT saddle-point free energy, as in [24, 25, 27, 29], equivalent to assuming that the chemical potentials are small relative to the temperature.

As we saw above, the eigenvalues $E_{I}^{(k)}$ scale as $\ell^{k+1}$ in the thermodynamic limit. Therefore, we shall rescale the chemical potentials as $\eta_{k} \rightarrow \frac{1}{\ell^{k}} \eta_{k}$, such that all the charges contribute at the same order in $\ell$ in the GGE partition function. With respect to the semiclassical limit of the $\mathrm{qBO}_{2}$ eigenvalues, the primary part scales as $b^{-k-1}$ and the 
descendant part scales as $b^{-k+1}$ (or order one and order $b^{2}$ after an overall rescaling by $\left.b^{k+1}\right)$. Therefore, at leading order in $c$, the GGE partition function depends only on the primary parts. In particular, if we set $a=0$ the saddle-point calculation gives the qKdV result $[24,25,27,29]$, as $E^{(2 n)}=0$ and $E^{(2 n+1)} \sim \Delta^{n+1}$. In the following, we present the saddle-point computation including the $\mathrm{U}(1)$ charge with the leading $b^{2} \approx 1 / c$ correction.

In the thermodynamic semiclassical limit, the GGE partition function (3.6) can be approximated by

$$
Z(\boldsymbol{\eta})=\int d \Delta d a e^{\mathcal{L}(\Delta, a, \boldsymbol{\eta})}=e^{F(\boldsymbol{\eta})}
$$

with

$$
\mathcal{L}=2 \pi \sqrt{\frac{c}{6} \Delta}+\eta a-\frac{\beta}{\ell}\left(\Delta+a^{2}\right)+\frac{\eta_{2}}{\ell^{2}}\left(a \Delta+\frac{1}{3} a^{3}\right)+\frac{\eta_{3}}{\ell^{3}}\left(\Delta^{2}+6 a^{2} \Delta+a^{4}\right)+\ldots
$$

Notice that we rotated $\eta \rightarrow i \eta$ and rescaled the chemical potentials with respect to $\ell$, as discussed in the previous subsection. Furthermore, similarly to [24], we rescale the parameters in (3.23) as

$$
a=\frac{\pi \ell}{b \beta} \alpha, \quad P=\frac{i \pi \ell}{b \beta} p \quad \Rightarrow \quad \Delta \sim\left(\frac{\pi \ell}{b \beta} p\right)^{2}
$$

and thus

$$
\mathcal{L}^{(0)} \equiv \frac{\mathcal{L}}{f_{0}}=2 p+\tilde{\eta} \alpha-\left(p^{2}+\alpha^{2}\right)+\tilde{\eta}_{2}\left(\alpha p^{2}+\frac{1}{3} \alpha^{3}\right)+\tilde{\eta}_{3}\left(p^{4}+6 \alpha^{2} p^{2}+\alpha^{4}\right)+\ldots
$$

where we defined

$$
f_{0}=\frac{\pi^{2} \ell}{\beta b^{2}}, \quad \tilde{\eta}=\frac{b}{\pi} \eta, \quad \tilde{\eta}_{k}=\left(\frac{\pi}{b}\right)^{k-1} \frac{\eta_{k}}{\beta^{k}}, k>1
$$

In the following, we shall consider the free energy as a function of the $\tilde{\eta}_{k}$ instead of $\eta_{k}$, consistent with our assumptions on the thermodynamic semiclassical limit. Notice that the overall constant $f_{0}$ in (3.25) is just (3.16) in the semiclassical limit $b \rightarrow 0$ and we used (2.4) to approximate

$$
\sqrt{\frac{c}{6}}=\frac{1}{b} \sqrt{1+\frac{13}{6} b^{2}+b^{4}} \approx \frac{1}{b} .
$$

We want to find the extrema of (3.25) from

$$
\left.\partial_{p} \mathcal{L}\right|_{p_{*}, \alpha_{*}}=0,\left.\quad \partial_{\alpha} \mathcal{L}\right|_{p_{*}, \alpha_{*}}=0
$$

and, if possible, those points that are minima of the free energy, which requires the determinant of the Hessian matrix to be positive. For generic values of the chemical potentials and domain of the variables, there is no global minima. However, it is still possible to find minima if we stick to a perturbative solution close to the CFT saddle-point. In the following, let us consider two cases with only $\eta_{2}$ and $\eta_{3}$ being non-zero, discarding all the other chemical potentials. 
First, suppose that $\tilde{\eta}_{k}=0$ for $k>2$ in (3.25). For $\alpha$ being a real number, $\mathcal{L}$ is clearly not bounded, as the highest order monomial is $\alpha^{3}$, but we shall press on and find the extrema. Solving for $p$ in (3.28), we get

$$
p_{*}=\frac{1}{1-\tilde{\eta}_{2} \alpha_{*}} .
$$

Plugging into the equation for $\alpha_{*}$ gives a quartic polynomial

$$
\tilde{\eta}_{2}^{3} \alpha_{*}^{4}-4 \tilde{\eta}_{2}^{2} \alpha_{*}^{3}+\tilde{\eta}_{2}\left(5+\tilde{\eta} \tilde{\eta}_{2}\right) \alpha_{*}^{2}-2\left(1+\tilde{\eta} \tilde{\eta}_{2}\right) \alpha_{*}+\tilde{\eta}+\tilde{\eta}_{2}=0
$$

To analyze how many real roots we have, in general we need to consider the discriminant of this polynomial. But if we expand the roots for small $\tilde{\eta}_{2}$, there are two complex roots, which we discard, and two real roots, one of which blows up as $\tilde{\eta}_{2}$ goes to zero. Choosing the only regular real root in this limit, we get the free energy

$$
F=f_{0}\left[1+\frac{\tilde{\eta}^{2}}{4}+\frac{1}{24}\left(12 \tilde{\eta}+\tilde{\eta}^{3}\right) \tilde{\eta}_{2}+\frac{1}{64}\left(16+24 \tilde{\eta}^{2}+\tilde{\eta}^{4}\right) \tilde{\eta}_{2}^{2}+\mathcal{O}\left(\tilde{\eta}_{2}^{3}\right)\right]
$$

Notice that if $\tilde{\eta}_{2}=0$ above, we recover the result (3.16).

Now, if we assume in addition that $\tilde{\eta}_{3}>0$, there should be at least one global minimum for $\mathcal{L}$ as the highest powers of $\alpha$ and $p$ in $\mathcal{L}$ are even. There are four roots for $p$ and $\alpha$, but only one pair is real and regular for small $\tilde{\eta}_{2}$ and $\tilde{\eta}_{3}$. Their expansion up to 2 nd order is

$$
\begin{aligned}
p_{*}= & 1+\frac{\tilde{\eta}}{2} \tilde{\eta}_{2}+\left(2+\frac{3}{2} \tilde{\eta}^{2}\right) \tilde{\eta}_{3}+\left(1+\frac{3 \tilde{\eta}^{2}}{4}\right) \frac{\tilde{\eta}_{2}^{2}}{2}+\left(10 \tilde{\eta}+\frac{5}{2} \tilde{\eta}^{3}\right) \tilde{\eta}_{2} \tilde{\eta}_{3}+ \\
& +\left(12+30 \tilde{\eta}^{2}+\frac{15}{4} \tilde{\eta}^{4}\right) \tilde{\eta}_{3}^{2}+\ldots, \\
\alpha_{*}= & \frac{\tilde{\eta}}{2}+\frac{1}{2}\left(1+\frac{1}{4} \tilde{\eta}^{2}\right) \tilde{\eta}_{2}+\left(3 \tilde{\eta}+\frac{1}{4} \tilde{\eta}^{3}\right) \tilde{\eta}_{3}+\frac{1}{4}\left(3 \tilde{\eta}+\frac{1}{4} \tilde{\eta}^{3}\right) \tilde{\eta}_{2}^{2}+ \\
& +\left(5+\frac{15}{2} \tilde{\eta}^{2}+\frac{5}{16} \tilde{\eta}^{4}\right) \tilde{\eta}_{2} \tilde{\eta}_{3}+\left(30 \tilde{\eta}+15 \tilde{\eta}^{3}+\frac{3}{8} \tilde{\eta}^{5}\right) \tilde{\eta}_{3}^{2}+\ldots,
\end{aligned}
$$

and the free energy expansion is then given by

$$
\begin{aligned}
\frac{F}{f_{0}}= & 1+\frac{\tilde{\eta}^{2}}{4}+\left(\frac{\tilde{\eta}}{2}+\frac{\tilde{\eta}^{3}}{24}\right) \tilde{\eta}_{2}+\left(1+\frac{3 \tilde{\eta}^{2}}{2}+\frac{\tilde{\eta}^{4}}{16}\right) \frac{\tilde{\eta}_{2}^{2}}{4}+ \\
& +\left[1+\frac{3 \tilde{\eta}^{2}}{2}+\frac{\tilde{\eta}^{4}}{16}+\left(5 \tilde{\eta}+\frac{5 \tilde{\eta}^{3}}{2}+\frac{\tilde{\eta}^{5}}{16}\right) \tilde{\eta}_{2}+\left(7+\frac{105}{4}\left(\tilde{\eta}^{2}+\frac{\tilde{\eta}^{4}}{8}\right)+\frac{7 \tilde{\eta}^{6}}{64}\right) \frac{\tilde{\eta}_{2}^{2}}{2}\right] \tilde{\eta}_{3}+ \\
& +\left[4+15 \tilde{\eta}^{2}+\frac{15 \tilde{\eta}^{4}}{4}+\frac{\tilde{\eta}^{6}}{16}+\left(56 \tilde{\eta}+70 \tilde{\eta}^{3}+\frac{21 \tilde{\eta}^{5}}{2}+\frac{\tilde{\eta}^{7}}{8}\right) \tilde{\eta}_{2}+\right. \\
& \left.+\left(45+315 \tilde{\eta}^{2}+\frac{1575 \tilde{\eta}^{4}}{8}+\frac{315 \tilde{\eta}^{6}}{16}+\frac{45 \tilde{\eta}^{8}}{256}\right) \tilde{\eta}_{2}^{2}\right] \tilde{\eta}_{3}^{2}+\ldots
\end{aligned}
$$


If we include all the chemical potentials in the partition function, we can in principle find a general solution in perturbative form

$$
\begin{aligned}
& p_{*}=1+\sum_{N=2}^{\infty} \sum_{m_{2}, m_{3}, \ldots, m_{N}=2} p_{m_{2}, m_{3}, \ldots, m_{N}}(\tilde{\eta}) \tilde{\eta}_{m_{2}} \tilde{\eta}_{m_{3}} \ldots \tilde{\eta}_{m_{N}}, \\
& \alpha_{*}=\frac{\tilde{\eta}}{2}+\sum_{N=2}^{\infty} \sum_{m_{2}, m_{3}, \ldots, m_{N}=2} \alpha_{m_{2}, m_{3}, \ldots, m_{N}}(\tilde{\eta}) \tilde{\eta}_{m_{2}} \tilde{\eta}_{m_{3}} \ldots \tilde{\eta}_{m_{N}} \\
& \frac{F}{f_{0}}=1+\frac{\tilde{\eta}^{2}}{4}+\sum_{N=2}^{\infty} \sum_{m_{2}, m_{3}, \ldots, m_{N}=2} f_{m_{2}, m_{3}, \ldots, m_{N}}(\tilde{\eta}) \tilde{\eta}_{m_{2}} \tilde{\eta}_{m_{3}} \ldots \tilde{\eta}_{m_{N}} .
\end{aligned}
$$

We could not find the generic expansion coefficients here as in [25], as we would need to know the primary energies for any $k$ and those have a more complicated form than the qKdV ones. For the particular case where only $\tilde{\eta}_{2}$ and $\tilde{\eta}_{3}$ are non-zero, if we substitute such series solutions into (3.28), we find that all coefficients are positive if all the chemical potentials are positive in our conventions. This in particular implies that the free energy is positive and bounded from below. The same computation is valid for the anti-holomorphic sector, as the eigenvalues are all real in this case.

\subsection{Leading quantum correction}

To calculate the free energy with the leading quantum correction, we need to consider the sum over descendants in the partition function

$$
Z(\boldsymbol{\eta})=\sum_{I} \int d p d \alpha e^{\mathcal{L}_{I}(p, \alpha, \boldsymbol{\eta})} \times(\text { anti-holo })=e^{F(\boldsymbol{\eta})},
$$

where anti-holo represents the anti-holomorphic sector. The perturbative expansion of $\mathcal{L}_{I}$ is of the form

$$
\frac{\mathcal{L}_{I}}{f_{0}}=\mathcal{L}^{(0)}+b^{2}\left(\mathcal{L}_{I}^{(1)}+\frac{13}{6} p\right)+\mathcal{O}\left(b^{4}\right)
$$

where $\mathcal{L}^{(0)}$ is defined in $(3.25)$ and only the subleading correction $\mathcal{L}_{I}^{(1)}$ depends on the descendant part. Notice that we also considered the contribution from (3.27) in the subleading correction. This is equivalent to the leading correction in the central charge expansion as $\frac{6}{c} \approx b^{2}$ for $b$ small.

As above, we will consider only $\eta_{2}$ and $\eta_{3}$ as being non-zero. The first $\mathrm{qBO}_{2}$ eigenvalue is given by

$$
-\frac{\beta}{\ell f_{0}} E_{I}^{(1)} \sim-\left(p^{2}+\alpha^{2}\right)-b^{2} \tilde{N}
$$

where we assumed the scaling

$$
N \sim\left(\frac{\pi \ell}{\beta}\right)^{2} \tilde{N}
$$


whose dependence on $\pi$ and $\beta$ should also be considered in the scalings (3.18). The semiclassical limit of the Calogero energies (2.26) is easily obtained using (3.18) and (3.24)

$$
\begin{aligned}
h_{\lambda}^{(k)}(P, a) & \approx b^{1-k} \sum_{j>0}\left[\left(b(P-i a)+\lambda_{j}\right)^{k}-(b(P-i a))^{k}\right] \\
& =b^{1-k} \sum_{j>0} \sum_{n=0}^{k-1}\left(\begin{array}{l}
k \\
n
\end{array}\right) \lambda_{j}^{k-n}(b(P-i a))^{n} \\
& \sim b^{2}\left(\frac{\pi \ell}{b \beta}\right)^{k+1} \sum_{m>0}\left[(i(p-\alpha)-m)^{k}-(i(p-\alpha))^{k}\right] \tilde{N}_{m},
\end{aligned}
$$

where we used the binomial expansion in the second line and the thermodynamic scaling in the last line. Considering the full Calogero contribution (2.25), we get

$$
\begin{array}{r}
\frac{\eta_{k}}{f_{0} \ell^{k}} e_{I}^{(k)} \sim b^{2} \tilde{\eta}_{k} \sum_{m}\left\{\left[(m+i(p-\alpha))^{k}-(i(p-\alpha))^{k}\right] \tilde{N}_{1, m}+\right. \\
\left.\left[(m-i(p+\alpha))^{k}-(-i(p+\alpha))^{k}\right] \tilde{N}_{2, m}\right\},
\end{array}
$$

which we conjecture should be valid for any $k$. Thus, including all the terms (3.40), we get

$$
\mathcal{L}_{I}^{(1)}=\sum_{j=1,2} \sum_{m>0} \sum_{k>0} d_{k} \tilde{\eta}_{k}\left[\left(m+i\left(\sigma_{j} p-\alpha\right)\right)^{k}-\left(i\left(\sigma_{j} p-\alpha\right)\right)^{k}\right] \tilde{N}_{j, m},
$$

with $\left(\sigma_{1}, \sigma_{2}\right)=(1,-1)$ and $d_{k}$ being constants. The constants we know explicitly are $\left(d_{1}, d_{2}, d_{3}\right)=(1, i / 2,-2)$.

We want to calculate the free energy again using the saddle-point approximation, but now the descendant part also depends on $p$ and $\alpha$. We can use the leading semiclassical saddle-point $\left(p_{*}, \alpha_{*}\right)$ to calculate the free energy up to $b^{2}$ order due to the following

$$
\begin{aligned}
\frac{1}{f_{0}} \mathcal{L}_{I}\left(p_{*}+b^{2} p_{1}, \alpha_{*}+b^{2} \alpha_{1}, \boldsymbol{\eta}\right)= & \mathcal{L}^{(0)}\left(p_{*}, \alpha_{*}, \tilde{\boldsymbol{\eta}}\right)+ \\
& +b^{2}\left(\mathcal{L}_{I}^{(1)}\left(p_{*}, \alpha_{*}, \tilde{\boldsymbol{\eta}}\right)+\frac{13}{6} p_{*}\right)+\mathcal{O}\left(b^{4}\right),
\end{aligned}
$$

where we used the saddle-point condition (3.28) to cancel the first correction to $\mathcal{L}^{(0)}$. Thus,

$$
Z \sim e^{f_{0}\left(\mathcal{L}^{(0)}\left(p_{*}, a_{*}, \tilde{\boldsymbol{\eta}}\right)+\frac{13 b^{2}}{6} p_{*}\right)} \sum_{I} e^{f_{0} b^{2} \mathcal{L}_{I}^{(1)}\left(p_{*}, \alpha_{*}, \tilde{\boldsymbol{\eta}}\right)} \times(\text { anti-holo }) .
$$

In general, the quantum correction has an imaginary part, but, as discussed in section 2.2.1, this can be canceled by considering the anti-holomorphic contribution to the partition function. Therefore, as we are considering a real saddle-point $\left(p_{*}, \alpha_{*}\right)$ and that all chemical potentials are real, we can simplify the quantum correction to

$$
\sum_{I} e^{f_{0} b^{2} \mathcal{L}_{I}^{(1)}\left(p_{*}, \alpha_{*}, \tilde{\boldsymbol{\eta}}\right)} \times(\text { anti-holo })=\sum_{\left\{\tilde{N}_{j, m}\right\}} e^{-\sum_{j, m} g_{j, m}\left(p_{*}, \alpha_{*}, \tilde{\boldsymbol{\eta}}\right) \tilde{N}_{j, m}},
$$


where $g_{j, m}$ is a real function obtained by cancelling the imaginary part of (3.41). In the particular case in which only the two first chemical potentials are non-zero, we have

$$
g_{j, m}=\frac{\pi^{2} \ell}{\beta}\left\{2 \tilde{\eta}_{3} m^{3}+\left[1+\left(\sigma_{j} p_{*}-\alpha_{*}\right) \tilde{\eta}_{2}-6\left(\sigma_{j} p_{*}-\alpha_{*}\right)^{2} \tilde{\eta}_{3}\right] m\right\}
$$

The sum over descendants in (3.44) can be done exactly via geometric series if we can guarantee that the sum is convergent, which requires the exponents to be positive for all $m$. For fixed $m$, the exponent is

$$
\begin{aligned}
g_{1, m} \tilde{N}_{1, m}+g_{2, m} \tilde{N}_{2, m}=\frac{\pi^{2} \ell}{\beta}\{ & {\left[2 \tilde{\eta}_{3} m^{3}+\left(1-\alpha_{*} \tilde{\eta}_{2}-6\left(p_{*}^{2}+\alpha_{*}^{2}\right) \tilde{\eta}_{3}\right) m\right]\left(\tilde{N}_{1, m}+\tilde{N}_{2, m}\right)+} \\
& \left.+p_{*}\left(\tilde{\eta}_{2}+12 \alpha_{*} \tilde{\eta}_{3}\right)\left(\tilde{N}_{1, m}-\tilde{N}_{2, m}\right)\right\} \\
= & \frac{\pi^{2} \ell}{\beta}\left\{2 \tilde{\eta}_{3} m^{3}+\left[1-6\left(p_{*}^{2}-\alpha_{*}^{2}\right) \tilde{\eta}_{3}\right] m\right\}\left(\tilde{N}_{1, m}+\tilde{N}_{2, m}\right),
\end{aligned}
$$

where we assumed in the second line that

$$
\tilde{\eta}_{2}+12 \alpha_{*} \tilde{\eta}_{3}=0
$$

to cancel the unbounded $\tilde{N}_{1, m}-\tilde{N}_{2, m}$ term. Furthermore, we assume that

$$
2 \tilde{\eta}_{3}\left[3\left(p_{*}^{2}-\alpha_{*}^{2}\right)-1\right] \leq 1
$$

for the remaining term in (3.46) to be positive under the condition $\tilde{\eta}_{3}>0$. The first condition (3.47) is incompatible with all the chemical potentials being positive, so we make $\tilde{\eta}_{2} \rightarrow-\tilde{\eta}_{2}$. However, in this case we cannot guarantee that $\alpha_{*}$ and the classical free energy $F$ are positive, as discussed before. We can check the conditions (3.47) and (3.48) at leading order in the chemical potentials

$$
\tilde{\eta}_{2}=-6 \tilde{\eta} \tilde{\eta}_{3}, \quad \tilde{\eta}_{3}\left[4-\frac{3}{2} \tilde{\eta}^{2}\right] \leq 1
$$

If the free energy is positive at this order, using the first equation in (3.49) we have

$$
\frac{F}{f_{0}} \approx 1+\frac{\tilde{\eta}^{2}}{4}+\left(1-\frac{3 \tilde{\eta}^{2}}{2}-\frac{9 \tilde{\eta}^{4}}{16}\right) \tilde{\eta}_{3} \geq 0
$$

For $\tilde{\eta}$ small enough such that

$$
4-\frac{3}{2} \tilde{\eta}^{2}>0, \quad 1-\frac{3 \tilde{\eta}^{2}}{2}-\frac{9 \tilde{\eta}^{4}}{16}>0
$$

we have at least one range compatible with the conditions above

$$
-\frac{1+\frac{\tilde{\eta}^{2}}{4}}{1-\frac{3 \tilde{\eta}^{2}}{2}-\frac{9 \tilde{\eta}^{4}}{16}}<0 \leq \tilde{\eta}_{3} \leq \frac{1}{4-\frac{3}{2} \tilde{\eta}^{2}} .
$$


Considering higher-order corrections in the chemical potentials complicates this analysis and requires a better knowledge of the saddle-point solutions. Assuming that convergence can be guaranteed by assumptions similar to the previous ones, we have

$$
\sum_{\left\{\tilde{N}_{j, m}\right\}} e^{-\sum_{j, m} g_{j, m}\left(p_{*}, \alpha_{*}, \tilde{\boldsymbol{\eta}}\right) \tilde{N}_{j, m}}=\prod_{j=1,2} \prod_{m=1}^{\infty} \frac{1}{1-e^{-g_{j, m}\left(p_{*}, \alpha_{*}, \tilde{\boldsymbol{\eta}}\right)}},
$$

and the free energy is thus given by

$$
F(\tilde{\boldsymbol{\eta}})=2 f_{0} \tilde{\mathcal{L}}^{(0)}(\tilde{\boldsymbol{\eta}})+\frac{13 \pi^{2} \ell}{3 \beta} p_{*}(\tilde{\boldsymbol{\eta}})-\sum_{j=1,2} \sum_{m>0} \log \left(1-e^{-\tilde{g}_{j, m}(\tilde{\boldsymbol{\eta}})}\right),
$$

where $\tilde{\mathcal{L}}^{(0)}(\tilde{\boldsymbol{\eta}}) \equiv \mathcal{L}^{(0)}\left(p_{*}(\tilde{\boldsymbol{\eta}}), \alpha_{*}(\tilde{\boldsymbol{\eta}}), \tilde{\boldsymbol{\eta}}\right)$ and $\tilde{g}_{j, m}(\tilde{\boldsymbol{\eta}}) \equiv g_{j, m}\left(p_{*}(\tilde{\boldsymbol{\eta}}), \alpha_{*}(\tilde{\boldsymbol{\eta}}), \tilde{\boldsymbol{\eta}}\right)$. This gives the free energy at the leading order in $1 / c$ considering both holomorphic and anti-holomorphic sectors.

There is another way to obtain a convergent descendant sum if we allow for a purely imaginary $a$. If we make the transformations $\tilde{\eta} \rightarrow i \tilde{\eta}, \tilde{\eta}_{2} \rightarrow i \tilde{\eta}_{2}$ and $\alpha_{*} \rightarrow i \alpha_{*}$, the resulting saddle-point values of $p_{*}, \alpha_{*}$ and $F$ are still real, although in this case not necessarily positive. The interesting point of this choice is that the terms with odd powers of $P$ in the descendant parts are cancelled, which mitigate part of the unboundedness problem we had in (3.46). In this case, the function $g_{j, m}$ results in

$$
g_{j, m}=\frac{\pi^{2} \ell}{\beta}\left\{2 \tilde{\eta}_{3} m^{3}+\frac{1}{2}\left(\tilde{\eta}_{2}+12 \alpha_{*} \tilde{\eta}_{3}\right) m^{2}+\left[1+\alpha_{*} \tilde{\eta}_{2}-6\left(p_{*}^{2}-\alpha_{*}^{2}\right) \tilde{\eta}_{3}\right] m\right\} .
$$

We could then analyze the positivity conditions for the descendant part and the free energy as above. However, there is not much conceptual gain of considering this case, so we will skip it here. Our conclusion is that the conditions to guarantee convergence of the partition function sum and the boundedness of the free energy are delicate when we include quantum corrections. The complete problem including all chemical potentials and quantum corrections shall be studied elsewhere.

\subsection{Free energy at finite $c$ with non-zero $\eta_{2}$}

We show now how to obtain the free energy from (3.22) at finite $c$ when only $\eta_{2}$ is non-zero, apart from $\beta$ and $\eta$ also non-zero. First, if we consider $\eta_{2}$ to be real, the real part of $E_{I}^{(2)}$ is given by (2.32). Therefore, summing the holomorphic and anti-holomorphic charges, we have in the thermodynamic limit

$$
\frac{1}{2 f_{0}} \mathcal{L}=2 p+\tilde{\eta} \alpha-\left(p^{2}+\alpha^{2}\right)+\tilde{\eta}_{2}\left(\alpha p^{2}+\frac{1}{3} \alpha^{3}\right)-b^{2} \sum_{j, m}\left[1-\tilde{\eta}_{2}\left(\alpha-\sigma_{j} p\right)\right] m \tilde{N}_{m}
$$

Defining

$$
\tau_{ \pm}\left(p, \alpha, \tilde{\eta}_{2}\right) \equiv \tau_{0}\left[1-\tilde{\eta}_{2}(\alpha \pm p)\right], \quad \tau_{0}=\frac{i \pi \ell}{\beta},
$$

it is clear that, if $\operatorname{Im} \tau_{ \pm}>0$, we can easily sum up the descendant part as geometric series

$$
\prod_{m=1}^{\infty}\left(\sum_{\tilde{N}_{m}=0}^{\infty} e^{2 \pi i \tau_{+} m \tilde{N}_{m}}\right) \prod_{n=1}^{\infty}\left(\sum_{\tilde{N}_{n}=0}^{\infty} e^{2 \pi i \tau_{-} m \tilde{N}_{n}}\right)=\prod_{n=1}^{\infty} \frac{1}{1-e^{2 \pi i \tau_{+} n}} \prod_{m=1}^{\infty} \frac{1}{1-e^{2 \pi i \tau_{-} m}}
$$


and thus

$$
\mathcal{L}=2 f_{0}\left[2 p+\tilde{\eta} \alpha-\left(p^{2}+\alpha^{2}\right)+\tilde{\eta}_{2}\left(\alpha p^{2}+\frac{1}{3} \alpha^{3}\right)\right]-\sum_{n=1}^{\infty} \log \left(1-e^{2 \pi i \tau_{+} n}\right)-\sum_{m=1}^{\infty} \log \left(1-e^{2 \pi i \tau_{-} m}\right)
$$

The next step to get the free energy is to do a saddle-point calculation, which is exact in the thermodynamic limit [24]. The resulting saddle-point equations (3.28) are

$$
\begin{aligned}
& \frac{1}{2 f_{0}} \partial_{p} \mathcal{L}=2\left(1-p+\tilde{\eta}_{2} \alpha p\right)-b^{2} \tilde{\eta}_{2}\left(\langle n\rangle_{+}-\langle n\rangle_{-}\right)=0 \\
& \frac{1}{2 f_{0}} \partial_{\alpha} \mathcal{L}=\tilde{\eta}-2 \alpha+\tilde{\eta}_{2}\left(p^{2}+\alpha^{2}\right)+b^{2} \tilde{\eta}_{2}\left(\langle n\rangle_{+}+\langle n\rangle_{-}\right)=0
\end{aligned}
$$

where we defined the descendant averages

$$
\langle n\rangle_{ \pm} \equiv\langle n\rangle\left(2 \pi i \tau_{ \pm}\left(p, \alpha, \tilde{\eta}_{2}\right)\right), \quad\langle f(n)\rangle(x) \equiv \sum_{n} \frac{f(n) e^{n x}}{1-e^{n x}}
$$

with $f(n)$ some arbitrary function of $n$. We can solve the system (3.60) perturbatively by Taylor expanding $p, \alpha$ and the descendant averages in $\tilde{\eta}_{2}$

$$
\begin{aligned}
p & =p_{0}+p_{1} \tilde{\eta}_{2}+\cdots, \\
\alpha & =\alpha_{0}+\alpha_{1} \tilde{\eta}_{2}+\cdots, \\
\langle n\rangle_{ \pm} & =\langle n\rangle_{0}-2 \pi i \tau_{0}\left(\alpha_{0} \pm p_{0}\right) \tilde{\eta}_{2}\langle n\rangle_{1}+\cdots
\end{aligned}
$$

with

$$
\langle n\rangle_{k}=\left.\partial_{x}^{k}\langle n\rangle\right|_{x=2 \pi i \tau_{0}}, \quad k \in \mathbb{N},
$$

being the connected correlators of $n$. Solving (3.60), we find the saddle-point values

$$
\begin{aligned}
& p_{*}(b)=p_{*}(0)+b^{2} \tilde{\eta}_{2}^{2}\left[\langle n\rangle_{0}-\frac{2 \pi^{2} \ell}{\beta}\langle n\rangle_{1}\right]+\mathcal{O}\left(\tilde{\eta}_{2}^{3}\right) \\
& \alpha_{*}(b)=\alpha_{*}(0)+b^{2}\left[\tilde{\eta}_{2}\langle n\rangle_{0}+\frac{1}{2} \tilde{\eta} \tilde{\eta}_{2}^{2}\left(\langle n\rangle_{0}+\frac{2 \pi^{2} \ell}{\beta}\langle n\rangle_{1}\right)\right]+\mathcal{O}\left(\tilde{\eta}_{2}^{3}\right),
\end{aligned}
$$

where $\left(p_{*}(0), \alpha_{*}(0)\right)$ are the classical values given in (3.32) with $\tilde{\eta}_{3}=0$. The total free energy is then

$$
\begin{aligned}
F(b)= & 2 F(0)-2 \sum_{n=1}^{\infty} \log \left(1-e^{-\frac{2 \pi^{2} \ell}{\beta} n}\right)+ \\
& +\frac{2 \pi^{2} \ell}{\beta}\left\{\tilde{\eta}\langle n\rangle_{0} \tilde{\eta}_{2}+\left[\left(\langle n\rangle_{0}+\frac{2 \pi^{2} \ell}{\beta}\langle n\rangle_{1}\right)\left(1+\frac{\tilde{\eta}^{2}}{4}\right)+b^{2}\langle n\rangle_{0}^{2}\right] \tilde{\eta}_{2}^{2}\right\}+\cdots
\end{aligned}
$$

where $F(0)$ is the classical value in $(3.33)$ with $\tilde{\eta}_{3}=0$. 
When $\tilde{\eta}_{2}$ is purely imaginary, the real eigenvalue is (2.31), and we can also do a finite $c$ saddle-point computation. In this particular case, the descendant sum does not depend on real $p$ and $\alpha$ and it is given by

$$
\begin{aligned}
& \sum_{(\lambda, \mu) \in \mathbb{Y}_{|\lambda|+|\mu|}} e^{-\frac{2 \beta}{\ell}(|\lambda|+|\mu|)-\frac{\eta_{2}}{\ell^{2}}\left(b^{-1} \sum_{j}\left(\lambda_{j}^{2}+\mu_{j}^{2}\right)+b \sum_{j}\left(\lambda_{j}^{\prime 2}+\mu_{j}^{\prime 2}\right)\right)}= \\
& \quad=\left[\sum_{\left\{\tilde{N}_{m}\right\}} e^{2 \pi i \tau_{0}\left(\sum_{m} a_{m} \tilde{N}_{m}+\frac{b^{2} \tilde{\eta}_{2}}{2} \sum_{n, m} \tilde{N}_{n} A_{n m} \tilde{N}_{m}\right)}\right]^{2}, \quad a_{m}=m+\frac{\tilde{\eta}_{2}}{2} m^{2},
\end{aligned}
$$

where we used the free boson representation identities derived in appendix $\mathrm{B}$ in the second line with the lower-triangular matrix $A_{n m}$ given by (B.9). The matrix $A$ is diagonalizable via $P^{t} \cdot A \cdot P=D$ with $D_{m n}=m \delta_{m n}$, as all the eigenvalues are distinct. Defining $\tilde{N}=P \cdot \tilde{N}^{\prime}$ and $a^{\prime}=a \cdot P$, we can thus simplify the expression inside brackets of (3.66) to

$$
\prod_{m=1}^{\infty} \sum_{\tilde{N}_{m}^{\prime}=0}^{\infty} e^{2 \pi i \tau_{0}\left(a_{m}^{\prime} \tilde{N}_{m}^{\prime}+b^{2} \frac{\tilde{\eta}_{2}}{2} m \tilde{N}_{m}^{\prime}{ }_{m}^{2}\right)}
$$

which is an infinite product of partial theta functions [76], as the sum goes from zero to infinity instead over all integers. Partial theta functions have also been obtained from characters of $\mathrm{W}$-algebras, in connection to false theta functions and, more generally, to quantum modular forms [77]. Although less studied than the usual theta functions, partial theta functions still have interesting quasi-modular properties and identities useful to study its asymptotics [78, 79]. We notice that the classical case $b=0$ of (3.67) has a descendant contribution given by

$$
\prod_{m=1}^{\infty} \frac{1}{1-e^{2 \pi i \tau_{0}\left(m+\frac{\tilde{n}_{2}}{2} m^{2}\right)}}
$$

The quadratic power of $m$ above is reminiscent of the Lifshitz free boson partition function discussed in [43], a non-relativistic correction to the CFT linear dispersion, which also has non-trivial quasi-modular properties. This partition function will be explored in more detail in a forthcoming work in connection to anisotropic field theories and non-relativistic CFTs.

\section{Generalized eigenstate thermalization for the $\mathrm{qBO}_{2}$ charges in the semiclassical limit}

In the previous section, we saw that the integrable structure of the $\mathrm{qBO}_{2}$ hierarchy allow us to describe a great deal of its GGE, including quantum corrections. Now we want to explore the Generalized ETH for the $\mathrm{qBO}_{2}$ charges, which is interpreted here as a comparison between matrix elements and ensemble averages of these charges.

First, let us briefly review the usual justification for why the ETH is equivalent to thermalization of a quantum many-body system. Given a Hamiltonian $H$ with no charged interactions, its orthonormal eigenbasis $\left|E_{n}, q\right\rangle$ with a commuting charge $q$, and an initial 
state $\left|\psi_{0}\right\rangle$, consider the time-evolved quantum state

$$
|\psi(t)\rangle=e^{-i H t}\left|\psi_{0}\right\rangle=\sum_{n, q} c_{n, q} e^{-i E_{n} t}\left|E_{n}, q\right\rangle, \quad c_{n, q}=\left\langle E_{n}, q \mid \psi_{0}\right\rangle
$$

The expectation value of an observable $\mathcal{O}$, which by assumption does not couple sectors of different charges, is given by

$$
\begin{aligned}
\langle\psi(t)|\mathcal{O}| \psi(t)\rangle= & \sum_{\substack{n, m, q \\
E_{n} \neq E_{m}}} e^{-i\left(E_{n}-E_{m}\right) t} c_{n, q} c_{m, q}^{*}\left\langle E_{m}, q|\mathcal{O}| E_{n}, q\right\rangle+ \\
& +\sum_{\substack{n, m, q \\
E_{n}=E_{m}}} c_{n, q} c_{m, q}^{*}\left\langle E_{m}, q|\mathcal{O}| E_{n}, q\right\rangle .
\end{aligned}
$$

Taking a time average of (4.2) over a period $\tau$, denoted by $\langle.\rangle_{\tau}$, and assuming there is no degeneracy in the spectrum, we get

$$
\langle\langle\psi(t)|\mathcal{O}| \psi(t)\rangle\rangle_{\tau}=\sum_{n, q}\left|c_{n, q}\right|^{2}\left\langle E_{n}, q|\mathcal{O}| E_{n}, q\right\rangle,
$$

the so-called diagonal ensemble. This ensemble strongly depends on the initial state via the coefficients $c_{n}$, and thus there is no apparent thermalization. The ETH is an assumption over the statistical distribution of matrix elements over the energy ensemble, making the diagonal ensemble equivalent to the microcanonical one in the thermodynamic limit. Thus, the many-body ETH can be stated as

$$
\left\langle E_{n}, q|\mathcal{O}| E_{m}, q\right\rangle=\mathcal{O}_{\mathrm{th}}(\bar{E}, q) \delta_{n m}+e^{-S(\bar{E}, q)} f_{\mathcal{O}}\left(E_{n}, E_{m}, q\right) R_{n m},
$$

where $\bar{E} \equiv\left(E_{n}+E_{m}\right) / 2, \mathcal{O}_{\text {th }}$ is a thermal average, $S$ the entropy, $f_{\mathcal{O}}$ is a slowly varying function and $R_{n m}$ is a random matrix of zero mean and unit variance [3, 4, 80]. The equivalence between the ensembles is strictly true in the thermodynamic limit, given that $S$ is an extensive quantity. The ETH might seem artificial in this presentation, but there are strong arguments that it should be valid for chaotic, non-integrable isolated quantum systems.

A more refined formulation of ETH is to consider the initial states as a result of a non-integrable, random perturbation over unperturbed energy eigenstates. Under certain reasonable assumptions, one can then derive the ETH form (4.4) [3]. An alternative formulation of (4.4) in the thermodynamic limit is that the diagonal matrix elements are given by smooth functions of the energy and charge [6]

$$
\left\langle E_{n}, q|\mathcal{O}| E_{n}, q\right\rangle=\mathcal{O}_{\text {eth }}\left(E_{n}, q\right) .
$$

For an integrable system, the eigenstates can depend on an infinite set of conserved charges $Q_{k}$. In the CFT case, all the higher charges can be written in terms of the energy and $\mathrm{U}(1)$ charge. Therefore, the Generalized ETH in the thermodynamic limit can be formulated as [29]

$$
\left\langle E_{n}, q|\mathcal{O}| E_{n}, q\right\rangle=\mathcal{O}_{\text {geth }}\left(Q_{k}\left(E_{n}\right)\right) .
$$

A fundamental question of thermalization is to answer if the proposed smooth functions are equivalent to an ensemble average. Here, we will compare $\mathcal{O}_{\text {geth }}$ with the related GGE 
ensemble average for the $\mathrm{qBO}_{2}$ charges. As discussed in the introduction, while this analysis might be relevant to verify the ensemble equivalence for operators in the vacuum family of holographic CFTs, it does not address the stronger version of generalized ETH for generic operators in 2D CFTs, which remains an open problem. Therefore, every mention on ETH below should be interpreted in the context of ensemble equivalence of conserved charges and operators in the vacuum family.

In this work, we are interested in the ETH for charged CFTs. Previous works noticed that the standard ETH is valid only for primary states in the strict large central charge limit $[6,7]$, with $1 / c$ corrections breaking the equality between ensembles. In another development, Calabrese and Cardy studied perturbations of 2D CFTs under a quantum quench [81], concluding that the now called Calabrese-Cardy states thermalize to the usual Gibbs ensemble. Later Cardy studied more generic states, perturbed by irrelevant deformations built from powers and derivatives of the energy-momentum tensor, and concluded that those thermalize to a non-abelian GGE, with conserved charges not necessarily commuting among themselves [18]. The qKdV GGE is formed by taking only a commuting subset of these more generic conserved charges [15]. The ETH failure for descendants and under the inclusion of $1 / c$ corrections, added to the observations that perturbed CFT states depend on higher conserved charges, justify the investigation of GGEs in 2D CFTs. The qKdV GGE was studied in [24-27] and the Generalized ETH for this case was proposed in [29]. There are some subtleties to be considered in the charged ETH when sectors with different charges interact [4]. However, here we are interested in the Generalized ETH for the subset of $\mathrm{qBO}_{2}$ charges in $\mathrm{U}(1)$ charged 2D CFTs. Although those are $\mathrm{U}(1)$ charged operators, they are diagonal in the AFLT basis and do not have interaction terms coupling distinct charged sectors. Therefore, we can consider the standard ETH condition as formulated above for the $\mathrm{qBO}_{2}$ charges.

Let $|P, a\rangle$ be a normalized primary eigenstate. The diagonal elements of the charges are

$$
\left\langle P, a\left|Q_{k}\right| P, a\right\rangle=\frac{E^{(k)}(P, a)}{\ell^{k}}
$$

and the strict Generalized ETH is valid if, in the thermodynamic limit,

$$
\left\langle Q_{k}\right\rangle_{\mathrm{GGE}}=\partial_{\eta_{k}} F=\frac{E^{(k)}(P, a)}{\ell^{k}} .
$$

This comparison is a first step to analyze the Generalized ETH for the vacuum Verma module, relevant to the $\mathrm{AdS}_{3} / \mathrm{CFT}_{2}$ correspondence [29]. This requires a more detailed study of the structure of quasi-primary operators for charged CFTs, to be investigated in a future work.

Although we do not have the GGE free energy in closed form for finite $c$, we can still check if the Generalized ETH for the charges can be satisfied in the thermodynamic semiclassical limit using the results of the previous section. We want to fix the chemical potentials to make eq. (4.8) true for any values of $P$ and $a$. To leading order in the semiclassical limit, the free energy is exactly given by its the saddle-point value

$$
F(\boldsymbol{\eta})=\mathcal{L}\left(P_{*}, a_{*}\right)=2 \pi \sqrt{\frac{c \Delta_{*}}{6}}+\sum_{k \geq 0} \frac{\eta_{k}}{\ell^{k}} E^{(k)}\left(P_{*}, a_{*}\right) .
$$


This implies that the GETH condition (4.8) is automatically satisfied in the thermodynamic, semiclassical limit if $P=P_{*}(\tilde{\boldsymbol{\eta}})$ and $a=a_{*}(\tilde{\boldsymbol{\eta}})$. Given that the saddle-point values are functions of the chemical potentials, we are fixing two of them to match the arbitrary $P$ and $a$ (typically the inverse temperature $\beta$ and the chemical potential $\eta$ ). All the other charges automatically match as they are polynomials of $P$ and $a$. This is equivalent to what happens in the qKdV hierarchy [24, 25].

Now let us consider the leading quantum correction to the free energy in (3.54). It is tempting to make the same choice as before, $P=P_{*}, a=a_{*}$, such that

$$
\begin{aligned}
\left\langle Q_{k}\right\rangle_{\mathrm{GGE}}-\frac{E^{(k)}(P, a)}{\ell^{k}} & \sim \frac{13 \pi^{2} \ell}{6} \partial_{\eta_{k}}\left(\frac{p_{*}}{\beta}\right)-\sum_{j=1,2} \sum_{m>0} \partial_{\eta_{k}} \log \left(1-e^{-\tilde{g}_{j, m}}\right) \\
& \approx \frac{13 \pi^{2} \ell}{6} \partial_{\eta_{k}}\left(\frac{p_{*}}{\beta}\right)-\sum_{j=1,2} \int_{0}^{\infty} d m \frac{\partial_{\eta_{k}} \tilde{g}_{j, m}}{e^{\tilde{g}_{j, m}}-1},
\end{aligned}
$$

where in the second step we replaced the sum over $m$ by an integral, which is a good approximation in the thermodynamic limit. Two chemical potentials have been fixed to make the zero order value of the charges to match and all the charges have a distinct subleading correction given by (4.10). Therefore, even if it is possible to separately cancel the corrections in principle, there is not enough chemical potentials to set all the subleading corrections to zero. This implies in a failure to match the GGE averages with primary state averages. On the other hand, we do not consider this a proof that the Generalized ETH does not work at $1 / c$ order, as the choice $P=P_{*}, a=a_{*}$ seems to be a particular ansatz. To achieve a concrete proof of this matter requires a better understanding of the quantum corrections and of the analytic structure of the saddle-point. Thus, we leave the analysis of the validity of the charged Generalized ETH including quantum corrections for the future.

\section{Discussion}

In this work, we presented the first steps to calculate the $\mathrm{qBO}_{2}$ GGE averages for charged 2D CFTs using the AGT correspondence. While our setup was rather general, our concrete calculations focused in the case in which only the first two non-trivial charges $Q_{2}$ and $Q_{3}$ were non-zero. The crucial ingredient to compute the GGE partition function was the AFTL basis, an alternative basis for descendants of the Virasoro and U(1) algebras, which diagonalizes all the $\mathrm{qBO}_{2}$ charges. The associated eigenvalues are generically complex, being a caveat to the equilibrium ensemble interpretation for chiral CFTs. However, those can make sense for non-chiral CFTs if we sum the holomorphic and anti-holomorphic charges, giving a real total contribution. While writing the $\mathrm{qBO}_{2}$ GGE partition function, we argued that the trace is equivalent to the case in which the Virasoro and $\mathrm{U}(1)$ modes do not commute, being the usual convention in the literature.

From the $\mathrm{qBO}_{2}$ GGE partition, we obtained the free energy in the thermodynamic semiclassical limit, including the leading quantum correction in the Liouville parameter $b$, equivalent to the leading correction in the central charge. Focusing on the first two nontrivial eigenvalues, we obtained the perturbative saddle-point solutions and discussed the 
conditions for the partition function convergence and boundedness of the free energy. We also presented the saddle-point analysis at finite $c$ with only $\eta_{2}$ being non-zero. For $\eta_{2}$ being real, we obtained a perturbative expansion of the free energy depending on descendant averages. For purely imaginary $\eta_{2}$, we obtained the exact descendant sums in the partition function in terms of partial theta functions. This example entails the main technical point of this paper, an alternative approach to obtain finite $c$ GGE averages using the AFLT basis.

In the last part of this paper, we briefly discussed the Generalized ETH for the $\mathrm{qBO}_{2}$ charges in the semiclassical limit, which is definitely obeyed at leading order in the central charge, just like the qKdV case. Whilst we argued that the Generalized ETH is not valid when $1 / c$ corrections are included, we deemed the argument preliminary as it is unclear how much it relies on the particular ansatz we made. We shall thus leave the appropriate analysis of the Generalized ETH of charged CFTs for a future work. For recent results on the ETH for charged CFTs, see [4, 80]. We emphasize that the analysis presented here should be relevant to the generalized ETH for the vacuum family in the semiclassical limit, but it should have limited application in the generalized ETH for generic heavy operators, whose averaged version without extra conserved charges is discussed in [12, 13].

There are many interesting forthcoming directions to our work. First, our analysis could be adapted to the qILW 2 hierarchy, whose spectrum has been conjectured in [36] and interpolates between the $\mathrm{qBO}_{2}$ and the $\mathrm{qKdV}$ plus free boson one. Therefore, although technically challenging, the exact $\mathrm{qKdV}$ spectrum and GGE partition function can be obtained by taking the appropriate limit of the $\mathrm{qILW}_{2}$ results. Another interesting direction is to obtain the qKdV GGE via the ODE/IQFT correspondence, as suggested in [82], which obtained the first few qKdV eigenvectors, the analogous of the AFLT basis in this case. It would also be intriguing to study the GGE with extra parafermionic or non-local charges, as suggested by $[18,83]$. The need to add quasi-local charges in the generalized ETH was observed in lattice integrable systems [84], leaving open the possibility that these charges might improve matters at finite $c$. Finally, as pointed out in our finite $c$ free energy computation, partial theta functions appear, which have modular properties related to false theta functions and quantum modular forms [77-79]. As we pointed out, these can be relevant in the studies of anisotropic field theories and non-relativistic CFTs [43] (see also [85]). In another development, [26] showed that correlation functions of qKdV charges have modular properties, obeying certain modular differential equations. It would also be worthwhile to investigate if the $\mathrm{qBO}_{2}$ correlation functions obey similar modular equations.

The natural holographic realization of the $\mathrm{qBO}_{2}$ hierarchy is $\mathrm{AdS}_{3}$ gravity with an extra $\mathrm{U}(1)$ gauge field, as described in [43], while the chiral case with only one $\mathrm{SL}(2) \times \mathrm{U}(1)$ current should be related to Warped CFTs (WCFT) [40, 86, 87], initially proposed as holographic duals of near-extremal black holes. We have seen that the $\mathrm{qBO}_{2}$ spectrum is not straightforwardly adapted to chiral CFTs, due to its complex eigenvalues, requiring further studies in the particular case of WCFTs and its related black hole solutions, in line with recent discussions in the $\mathrm{KdV}$ case [41, 45].

The results presented here can also be useful to explore thermalization in effective field theory descriptions of lattice systems, in which higher integrable charges can be interpreted as precise deformations of the 2D CFT description [88]. Along these lines, the quantum 
Benjamin- $\mathrm{Ono}_{2}$ equation has been proposed to describe non-linear deviations from the CFT description in the fractional Quantum Hall liquid [32, 46-48], suggesting a concrete physical system to apply our results.

\section{Acknowledgments}

I would like to thank stimulating discussions with Marcone Sena, David Tempo and Ricardo Troncoso about this work. The Centro de Estudios Científicos (CECs) is funded by the Chilean Government through the Centers of Excellence Base Financing Program of Conicyt.

\section{A Derivation of integrals of motion and analytic ordering}

Given two local operators $A(z)$ and $B(w)$, their operator product expansion have divergent terms that need to be regulated. The standard procedure to regularize this product in CFT is called analytic ordering, a generalization of the normal ordering for free fields [15, 61]. An algorithm to calculate the analytic ordering on the cylinder has been pedagogically described in [39]. Here we present a variation of that approach using OPEs and including $\mathrm{U}(1)$ terms with Hilbert transforms.

The main idea of analytic ordering is to make a Cauchy integral that removes all the singular parts in the coincidence limit $z \rightarrow w$. The analytic ordering of two operators $A(z)$ and $B(z)$ on the cylinder is then defined as

$$
(A B)(w)=\oint \frac{d z}{2 \pi i} \frac{1}{z-w} \mathcal{T}(A(z) B(w)),
$$

where

$$
\mathcal{T}(A(z) B(w))= \begin{cases}A(z) B(w), & \operatorname{Re} z<\operatorname{Re} w \\ B(w) A(z), & \operatorname{Re} z>\operatorname{Re} w\end{cases}
$$

is the time-ordering operator. This definition is the same for operators defined on the plane, but instead of time-ordering (real part of $z$ ), we use radial ordering. When expressed in terms of the $A$ and $B$ modes on the plane, the two ordering procedures differ. Our strategy here is to rewrite (A.1) in terms of coordinates and operators on the plane. In the following, we simply differ operators on the cylinder and on the plane by its coordinates: $A(z), B(w)$ for the former and $A(u), B(v)$ for the latter.

Any OPE can be expressed as a singular part and a regular part [61]

$$
A(u) B(v)=\overparen{A(u) B}(v)+(A(u) B(v)),
$$

where the singular part is

$$
A(u) B(v) \sim \overparen{A(u) B}(v)=\sum_{k>0} \frac{\{A B\}_{k}(v)}{(u-v)^{k}}
$$

and the regular part

$$
(A(u) B(v))=\sum_{k \geq 0} \frac{(u-v)^{k}}{k !}\left(\partial^{k} A B\right)(v) .
$$


The $\sim$ sign represents the display of only the singular part. If $A, B$ have holomorphic weights $h_{A}, h_{B}$ respectively, we expand the operators on the plane as

$$
A(u)=\sum_{n} A_{n} u^{-n-h_{A}}, \quad B(u)=\sum_{n} B_{n} u^{-n-h_{B}} .
$$

If we define

$$
(A B)(u)=\sum_{n}(A B)_{n} u^{-n-h_{A}-h_{B}},
$$

using the analytic ordering on the plane, we get [61]

$$
(A B)_{m}=\sum_{n \leq-h_{A}} A_{n} B_{m-n}+\sum_{n>-h_{A}} B_{m-n} A_{n}
$$

We can map the cylinder to the plane by a conformal transformation $u=e^{z}$. In general, primary fields transform as

$$
A(z)=\left(\frac{d z}{d u}\right)^{-h_{A}} A(u)=u^{h_{A}} A(u) \equiv \tilde{A}(u)
$$

where we remind that we distinguish the operator domains by their argument. Operators on the cylinder have a mode expansion around $u=0$ equivalent to an operator of zero weight on the plane

$$
\tilde{A}(u)=\sum_{n} \tilde{A}_{n} u^{-n}
$$

This is also the case of the energy-momentum tensor $T(z)$, even though it is a quasiprimary. We want to express the integral (A.1) in plane coordinates

$$
\frac{d z}{2 \pi i} \frac{u^{h_{A}} A(u) v^{h_{B}} B(v)}{z-w}=\frac{d u}{2 \pi i u} \frac{1}{\ln \left(1+\frac{u-v}{v}\right)} \tilde{A}(u) \tilde{B}(v) .
$$

The integrand can be rewritten in terms of the Bernoulli polynomials of the second kind via its generating function [89]

$$
\frac{(z+1)^{x}}{\ln (1+z)}=\sum_{n=0}^{\infty} \psi_{n}(x) z^{n-1}, \quad|z|<1 .
$$

The first five polynomials are

$$
\begin{gathered}
\psi_{0}(x)=1, \quad \psi_{1}(x)=x+\frac{1}{2}, \quad \psi_{2}(x)=\frac{1}{2} x^{2}-\frac{1}{12} \\
\psi_{3}(x)=\frac{1}{6} x^{3}-\frac{1}{2} x^{2}+\frac{1}{24}, \quad \psi_{4}(x)=\frac{1}{24} x^{4}-\frac{1}{6} x^{3}+\frac{1}{6} x^{2}-\frac{19}{720} .
\end{gathered}
$$

The simplest relevant values for us are $x=0$ and $x=1$

$$
\psi_{n}(0)=G_{n}, \quad \psi_{n}(1)=G_{n-1}+G_{n},
$$


where $G_{n}$ are the reciprocal logarithmic numbers or Gregory coefficients ${ }^{2}$ obtained from

$$
\frac{z}{\log (1+z)}=1+\frac{1}{2} z-\frac{1}{12} z^{2}+\frac{1}{24} z^{3}-\frac{19}{720} z^{4}+\cdots=1+\sum_{n=1}^{\infty} G_{n} z^{n} .
$$

Using (A.12) with $z=\frac{u-v}{v}$ and $x=0$ in (A.11), we get from (A.1)

$$
\begin{aligned}
(A B)(w) & =\oint_{v} \frac{d u}{2 \pi i u}\left(\frac{v}{u-v}+\sum_{n=1}^{\infty} \psi_{n}(0)\left(\frac{u-v}{v}\right)^{n-1}\right) \tilde{A}(u) \tilde{B}(v) \\
& =\left(\tilde{A}_{-} \tilde{B}_{+}\right)(v)+\oint_{v} \frac{d u}{2 \pi i} \sum_{n=1}^{\infty} \psi_{n}(0)\left(\frac{u-v}{v}\right)^{n-1} u^{h_{A}-1} v^{h_{B}} \sum_{k=1}^{h_{A}+h_{B}} \frac{\{A B\}_{k}(v)}{(u-v)^{k}} .
\end{aligned}
$$

The first term above is the analytic ordering on the plane of the operators defined as

$$
\tilde{A}_{ \pm}(u)=\sum_{n} \tilde{A}_{n} u^{-n \pm 1}
$$

The modes of $\left(\tilde{A}_{-} \tilde{B}_{+}\right)(v)$ are given by (A.8) with $h_{\tilde{A}_{-}}=1$

$$
\begin{aligned}
\left(\tilde{A}_{-} \tilde{B}_{+}\right)_{m} & =\sum_{n \leq-1} \tilde{A}_{n} \tilde{B}_{m-n}+\sum_{n>-1} \tilde{B}_{m-n} \tilde{A}_{n} \\
& =\sum_{n \geq 1}\left(\tilde{A}_{-n} \tilde{B}_{m+n}+\tilde{B}_{m-n} \tilde{A}_{n}\right)+\tilde{B}_{m} \tilde{A}_{0} .
\end{aligned}
$$

The second term depends only on the singular OPE, which can be computed with the methods described in [61]. As it stands, the OPE will produce derivatives of the function $u^{h_{A}-1}$. Assuming $h_{A} \geq 1$, we can Taylor expand this function around $u=v$

$$
u^{h_{A}-1}=v^{h_{A}-1} \sum_{p=0}^{h_{A}-1}\left(\begin{array}{c}
h_{A}-1 \\
p
\end{array}\right)\left(\frac{u-v}{v}\right)^{p},
$$

and then

$$
\begin{aligned}
& v^{h_{A}+h_{B}-1} \oint_{v} \frac{d u}{2 \pi i} \sum_{k=1}^{h_{A}+h_{B}} \sum_{n=1}^{\infty} \sum_{p=0}^{h_{A}-1}\left(\begin{array}{c}
h_{A}-1 \\
p
\end{array}\right) \psi_{n}(0)(u-v)^{n+p-k-1} v^{1-n-p}\{A B\}_{k}(v) \\
& =\sum_{k=1}^{h_{A}+h_{B}} \sum_{n=1}^{\infty} \sum_{p=0}^{h_{A}-1}\left(\begin{array}{c}
h_{A}-1 \\
p
\end{array}\right) \psi_{n}(0) \delta_{n+p, k} v^{h_{A}+h_{B}-k}\{A B\}_{k}(v) \\
& =\sum_{k=1}^{h_{A}+h_{B}}\left(\sum_{n=1}^{k}\left(\begin{array}{c}
h_{A}-1 \\
k-n
\end{array}\right) \psi_{n}(0)\right) v^{h_{A}+h_{B}-k}\{A B\}_{k}(v) \\
& =\sum_{k=1}^{h_{A}+h_{B}}\left(\psi_{k}\left(h_{A}-1\right)-\left(\begin{array}{c}
h_{A}-1 \\
k
\end{array}\right)\right) v^{h_{A}+h_{B}-k}\{A B\}_{k}(v),
\end{aligned}
$$

\footnotetext{
${ }^{2}$ These can be also easily obtained from $G_{n}=-\frac{B_{n}^{(n-1)}(0)}{(n-1) n !}$, where $B_{n}^{(s)}(z)$ are the Generalized Bernoulli polynomials or Norlund polynomials, implemented in Mathematica as NorlundB [n, s,z] .
} 
where in the fourth line we used the identity

$$
\sum_{n=1}^{k}\left(\begin{array}{c}
h_{A}-1 \\
k-n
\end{array}\right) \psi_{n}(0)=\psi_{k}\left(h_{A}-1\right)-\left(\begin{array}{c}
h_{A}-1 \\
k
\end{array}\right) .
$$

If we expand the OPE coefficients as

$$
\{A B\}_{k}(v)=\sum_{m}\{A B\}_{k, m} v^{-h_{A}-h_{B}+k-m},
$$

going back to (A.16), we get

$$
(A B)(w)=\sum_{m}\left[\left(\tilde{A}_{-} \tilde{B}_{+}\right)_{m}+\{A B\}_{m}\right] v^{-m}
$$

with

$$
\{A B\}_{m} \equiv \sum_{k=1}^{h_{A}+h_{B}}\left(\psi_{k}\left(h_{A}-1\right)-\left(\begin{array}{c}
h_{A}-1 \\
k
\end{array}\right)\right)\{A B\}_{k, m} .
$$

The OPE modes can be obtained from the inverse formula of (A.20)

$$
\{A B\}_{k, m}=\oint \frac{d v}{2 \pi i} v^{h_{A}+h_{B}-k+m-1}\{A B\}_{k}(v) .
$$

From this, the zero mode on the cylinder is given by

$$
\begin{aligned}
(A B)_{0} & =\oint \frac{d w}{2 \pi i}(A B)(w) \\
& =\left(\tilde{A}_{-} \tilde{B}_{+}\right)_{0}+\{A B\}_{0} .
\end{aligned}
$$

Therefore, we reduced the problem of analytic ordering to finding the coefficients of the OPEs between operators. We can recursively use the definitions above to find the $\mathrm{qBO}_{2}$ charges. Notice that the procedure needs to be slightly modified if the operators are descendants, i.e., involve derivatives of primaries, and for the energy-momentum tensor. We do not display a general formula for these cases, but we will show some examples below on how to proceed.

Let us apply the zero mode formula (A.24) for the simplest cases $J^{2}$ and $T^{2}$. The currents $T$ and $J$ change under $u=e^{z}$ as

$$
J(z)=u J(u)=\sum_{n} a_{n} u^{-n}, \quad T(z)=u^{2} T(u)-\frac{c}{24}=\sum_{n} \tilde{L}_{n} u^{-n},
$$

where

$$
\tilde{L}_{n}=L_{n}-\frac{c}{24} \delta_{n, 0}
$$

Thus, although $T$ is a quasiprimary, we can directly apply the formulas above for $T$ if we use the shifted modes in the first term of (A.24). The fundamental singular OPEs of $J$ and $T$ on the plane are

$$
J(u) J(v) \sim \frac{1 / 2}{(u-v)^{2}}, \quad T(u) J(v) \sim 0,
$$


and

$$
T(u) T(v) \sim \frac{c / 2}{(u-v)^{4}}+\frac{2 T(v)}{(u-v)^{2}}+\frac{\partial T(v)}{u-v} .
$$

From (A.8), we have that

$$
\left(\tilde{J}_{-} \tilde{J}_{+}\right)_{0}=\sum_{n \leq-1} a_{n} a_{-n}+\sum_{n>-1} a_{-n} a_{n}=2 \sum_{n>0} a_{-n} a_{n}+a_{0}^{2}
$$

From the definition (A.4), the $J J$ OPE (A.27) and the Bernoulli polynomials $\psi_{n}(0)$ obtained from (A.14) and (A.15), we find

$$
\left\{\tilde{J}_{-} \tilde{J}_{+}\right\}_{0}=\sum_{k=1}^{2} \psi_{k}(0)\{J J\}_{k, 0}=-\frac{1}{24},
$$

where we used that $\left(\begin{array}{l}0 \\ x\end{array}\right)=0, x>0$. The zero mode on the cylinder is thus the sum of (A.29) and (A.30)

$$
(J J)_{0}=2 \sum_{n>0} a_{-n} a_{n}+a_{0}^{2}-\frac{1}{24} .
$$

For the TT zero mode, we get

$$
\begin{aligned}
(T T)_{0} & =\sum_{n \leq-1} \tilde{L}_{n} \tilde{L}_{-n}+\sum_{n>-1} \tilde{L}_{-n} \tilde{L}_{n}+\sum_{k=1}^{4} \psi_{k}(1)\{T T\}_{k, 0}-\{T T\}_{1,0} \\
& =2 \sum_{n>1} L_{n} L_{-n}+\tilde{L}_{0}^{2}+\frac{11}{720} \frac{c}{2}+\frac{5}{12} 2 L_{0}-\frac{3}{2} 2 L_{0}+2 L_{0} \\
& =2 \sum_{n>0} L_{-n} L_{n}+L_{0}^{2}-\frac{c+2}{12} L_{0}+\frac{c^{2}}{(24)^{2}}+\frac{11 c}{1440} \\
& =2 \sum_{n>0} L_{-n} L_{n}+L_{0}^{2}-\frac{c+2}{12} L_{0}+\frac{5 c^{2}+22 c}{2880}
\end{aligned}
$$

which matches the known result [15, 39].

\section{A.1 Derivation of $Q_{2}$}

To complete our minimal set of examples, let us derive $Q_{2}$ in (2.23). The first term $T J$ is easy as its singular OPE is zero

$$
(T J)_{0}=\left(\tilde{T}_{-} \tilde{J}_{+}\right)_{0}=\sum_{n} \tilde{L}_{-n} a_{n}=\left[\sum_{n \neq 0} L_{-n} a_{n}+a_{0}\left(L_{0}-\frac{c}{24}\right)\right] .
$$

The third term is

$$
\left(J^{3}\right)_{0}=\left(J\left(J^{2}\right)\right)_{0}=\left[\sum_{m \leq-1} a_{m}\left(J^{2}\right)_{-m}+\sum_{m>-1}\left(J^{2}\right)_{-m} a_{m}\right]+\sum_{k>0} \psi_{k}(0)\left\{J\left(J^{2}\right)\right\}_{k, 0} .
$$


Using that

$$
\left(J^{2}\right)_{m}=\sum_{n \leq-1} a_{n} a_{m-n}+\sum_{n>-1} a_{m-n} a_{n}-\frac{1}{24} \delta_{m, 0},
$$

the first two terms inside brackets in (A.34) simplify to

$$
\begin{aligned}
& \sum_{m \leq-1} \sum_{n \leq-1} a_{m} a_{n} a_{-m-n}+\sum_{m \leq-1} \sum_{n>-1} a_{m} a_{-m-n} a_{n}+\sum_{m>-1} \sum_{n \leq-1} a_{n} a_{-m-n} a_{m}-\frac{1}{24} a_{0} \\
& +\sum_{m>-1} \sum_{n>-1} a_{-m-n} a_{n} a_{m}=\sum_{n+m+p=0}: a_{n} a_{m} a_{p}:-\frac{1}{24} a_{0},
\end{aligned}
$$

where :: denotes the usual normal ordering of placing the highest modes to the right. To obtain the singular OPE, we use the generalized Wick theorem [61]

$$
\overrightarrow{J(z)(J} J)(w)=\oint \frac{d x}{2 \pi i} \frac{1}{x-w}(\overrightarrow{J(z) J}(x) J(w)+J(x) \overrightarrow{J(z) J}(w))=\frac{J(w)}{(z-w)^{2}} .
$$

One can show by induction that

$$
\left.\overparen{J(z)\left(J^{n}\right.}\right)(w)=\frac{n}{2} \frac{\left(J^{n-1}\right)(w)}{(z-w)^{2}},
$$

which then implies that

$$
\left\{J\left(J^{n}\right)\right\}_{k, m}=\frac{n}{2}\left(J^{n-1}\right)_{m} \delta_{k, 2} .
$$

Going back to (A.34), we get

$$
\begin{aligned}
\left(J^{3}\right)_{0} & =\sum_{n+m+p=0}: a_{n} a_{m} a_{p}:-\frac{1}{24} a_{0}+\psi_{2}(0) a_{0} \\
& =\sum_{\substack{n+m+p=0 \\
n, m, p \neq 0}}: a_{n} a_{m} a_{p}:+a_{0}^{3}+3 a_{0}\left(2 \sum_{n>0} a_{-n} a_{n}-\frac{1}{24}\right)
\end{aligned}
$$

The second term in $Q_{2}$ is $\left(J \mathcal{H} \partial_{x} J\right)$ and requires us to deal with a derivative and the Hilbert transform (2.22). Let us define the Hilbert transform derivative operator

$$
\mathcal{D}_{u}^{H} \equiv i \mathcal{H} u \partial_{u}=\mathcal{H} \partial_{x},
$$

where we used that $u=e^{i x}$. The analytic continuation of the Hilbert transform on the circle $(2.22)$ can be written as

$$
\mathcal{H} f(u)=i \mathcal{P} \oint_{C} \frac{d \zeta}{2 \pi i} f(\zeta) \frac{\zeta+u}{\zeta(\zeta-u)},
$$

where the contour $C$ passes through $\zeta=u$ (which is why we need the principal value integral). To calculate (A.42) in practice, we use two simple results:

$$
\frac{\zeta+u}{\zeta(\zeta-u)}=\frac{2}{\zeta-u}-\frac{1}{\zeta}
$$


and

$$
2 \mathcal{P} \oint_{C}=\lim _{\epsilon \rightarrow 0}\left(\oint_{C_{+\epsilon}}+\oint_{C_{-\epsilon}}\right)
$$

where $C_{ \pm \epsilon}$ denotes a contour centered at the origin with radius $|u| \pm \epsilon$. The first essential property of the Hilbert transform for us is

$$
\mathcal{H} u^{n}=i \operatorname{sgn}(n) u^{n},
$$

such that

$$
\mathcal{D}_{u}^{H} u^{n}=-n \operatorname{sgn}(n) u^{n}=-|n| u^{n} .
$$

Given (A.45), we have

$$
\mathcal{D}_{u}^{H} \tilde{J}(u)=-\sum_{n}|n| a_{n} u^{-n} .
$$

This gives the first part of (A.24)

$$
\left(\tilde{J}_{-}\left(\mathcal{D}_{u}^{H} \tilde{J}\right)_{+}\right)_{0}=\sum_{n>0}\left[a_{-n}\left(|n| a_{n}\right)+\left(|-n| a_{-n}\right) a_{n}\right]=2 \sum_{n>0}|n| a_{-n} a_{n} .
$$

The OPE part of the analytic ordering requires an adaptation of its derivation, starting from (A.16). The structure of the integral is

$$
\oint_{v} \frac{d u}{2 \pi i} g(u, v) \tilde{J}(u) \mathcal{D}_{v}^{H} \tilde{J}(v)=\oint_{v} \frac{d u}{2 \pi i} g(u, v) \mathcal{D}_{v}^{H}(\tilde{J}(u) \tilde{J}(v))
$$

where

$$
g(u, v)=\frac{1}{u} \sum_{n=1}^{\infty} \psi_{n}(0)\left(\frac{u-v}{v}\right)^{n-1} .
$$

Then

$$
\begin{aligned}
\mathcal{D}_{v}^{H}(\tilde{J}(u) \vec{J}(x)) & =\mathcal{D}_{v}^{H}\left[\frac{u v / 2}{(u-v)^{2}}\right] \\
& =\frac{i u}{2} \mathcal{H}_{v}\left[\frac{v(u+v)}{(u-v)^{3}}\right] \\
& =-\frac{u v(u+v)}{2(u-v)^{3}} \operatorname{Sgn}(|u|-|v|) \\
& =-\left(\tilde{J}(u) v \partial_{v} \tilde{J}(v)\right) \operatorname{Sgn}(|u|-|v|),
\end{aligned}
$$

where in the last line we used

$$
\tilde{J} \widetilde{(u) v \partial_{v} \vec{J}}(v)=u v\left(\frac{1 / 2}{(u-v)^{2}}+\frac{v}{(u-v)^{3}}\right)=\frac{u v(u+v)}{2(u-v)^{3}} .
$$


The third line in (A.51) is a result of the following crucial property of the Hilbert transform. Let $f$ be a function with decomposition $f=f_{+}+f_{-}$, where $f_{+}$is analytic inside a disc $D$ and $f_{-}$is analytic outside $D$. The Hilbert transform of $f$ is given by

$$
\mathcal{H} f=i\left(f_{+}-f_{-}\right)
$$

and, in particular,

$$
\mathcal{H} f_{ \pm}= \pm i f_{ \pm}
$$

As the domain of analyticity of the OPE depends on the relative radius of the insertions, we get the result (A.51). Going back to (A.49) and using the decomposition

$$
\oint_{v}=\lim _{\epsilon \rightarrow 0}\left(\oint_{C_{|v|+\epsilon}}-\oint_{C_{|v|-\epsilon}}\right)
$$

we get

$$
\begin{aligned}
& -\oint_{v} \frac{d u}{2 \pi i} \frac{1}{u} \sum_{n=1}^{\infty} \psi_{n}(0)\left(\frac{u-v}{v}\right)^{n-1} \tilde{J} \widetilde{(u) v \partial_{v} \tilde{J}}(v) \operatorname{Sgn}(|u|-|v|) \\
& =-\sum_{k=2}^{3} \psi_{k}(0)\left\{J v \partial_{v} \tilde{J}\right\}_{k, m} \\
& =-\delta_{m, 0}\left(-\frac{1}{12} \times \frac{1}{2}+\frac{1}{24}\right)=0
\end{aligned}
$$

Therefore,

$$
\left(\tilde{J}(u) \mathcal{D}_{v}^{H} \tilde{J}(v)\right)_{0}=2 \sum_{n>0}|n| a_{-n} a_{n},
$$

and the final result for $Q_{2}$ is

$$
Q_{2}=\sum_{n \neq 0} L_{-n} a_{n}+2 i Q \sum_{n>0}|n| a_{-n} a_{n}+\frac{1}{3} \sum_{n+m+p=0}: a_{n} a_{m} a_{p}:+a_{0}\left(L_{0}-\frac{c+1}{24}\right) .
$$

One can also easily adapt the generalized Wick theorem for other OPEs and obtain higher charges. The coefficients of the $\mathrm{qBO}_{2}$ charges are fixed by explicitly computing the commutators of distinct charges. This computation also reduces to calculating OPEs of composite operators.

\section{A.2 Derivation of $Q_{3}$}

Here we briefly quote the regularized terms of the charge $Q_{3}$. The associated current is

$$
\mathcal{Q}_{4}=T^{2}+6 T J^{2}+6 i Q\left(T \mathcal{D}_{v}^{H} J+J^{2} \mathcal{D}_{v}^{H} J\right)-6 Q^{2}\left(\mathcal{D}_{v}^{H} J\right)^{2}+\left(1+Q^{2}\right) J_{x}^{2}+J^{4}
$$


The zero mode of each term is given by

$$
\begin{aligned}
\left(T^{2}\right)_{0} & =2 \sum_{n>0} L_{-n} L_{n}+L_{0}^{2}-\frac{c+2}{12} L_{0}+\frac{5 c^{2}+22 c}{2880}, \\
\left(T J^{2}\right)_{0} & =\sum_{m \neq 0} \sum_{n+p=m} L_{-m} a_{n} a_{p}+2\left(L_{0}-\frac{c}{24}\right) \sum_{n>0} a_{-n} a_{n}+\left(a_{0}^{2}-\frac{1}{24}\right)\left(L_{0}-\frac{c}{24}\right), \\
\left(T \mathcal{D}_{x}^{H} J\right)_{0} & =\sum_{n \neq 0}|n| L_{n} a_{-n}, \\
\left(J^{2} \mathcal{D}_{x}^{H} J\right)_{0} & =\sum_{m+n+p=0}|p|: a_{m} a_{n} a_{p}: \\
\left(\mathcal{D}_{x}^{H} J \mathcal{D}_{x}^{H} J\right)_{0} & =2 \sum_{n>0} n^{2} a_{-n} a_{n}+\frac{1}{240}, \\
\left(J_{x} J_{x}\right)_{0} & =-\left(u \partial_{u} J u \partial_{u} J\right)_{0}=2 \sum_{n>0} n^{2} a_{-n} a_{n}+\frac{1}{240}, \\
\left(J^{4}\right)_{0} & =\left(J\left(J^{3}\right)\right)_{0}=\sum_{m+n+p+q=0}: a_{m} a_{n} a_{p} a_{q}:-\frac{6}{24}\left(2 \sum_{n>0} a_{-n} a_{n}+a_{0}^{2}\right)+\frac{15}{2880} .
\end{aligned}
$$

The results for the terms only involving $J^{\prime} s$ can be also obtained from zeta function regularization. The sum of all these terms, after simplifications, give the charge

$$
\begin{aligned}
Q_{3}= & 2 \sum_{n>0} L_{-n} L_{n}+L_{0}^{2}-\frac{c+5}{12} L_{0}+\frac{5 c^{2}+42 c+37}{2880}+6 \sum_{m \neq 0} \sum_{n+p=m} L_{-m} a_{n} a_{p}+ \\
& 6\left(L_{0}-\frac{c+1}{24}\right)\left(2 \sum_{n>0} a_{-n} a_{n}+a_{0}^{2}\right)+6 i Q \sum_{n \neq 0}|n| L_{n} a_{-n}+ \\
& 6 i Q \sum_{m+n+p=0}|p|: a_{m} a_{n} a_{p}:+2\left(1-5 Q^{2}\right) \sum_{n>0} n^{2} a_{-n} a_{n}+\sum_{m+n+p+q=0}: a_{m} a_{n} a_{p} a_{q}: .
\end{aligned}
$$

This result slightly differs from [33] in the vacuum eigenvalue, if we set Virasoro and U(1) zero modes to zero. We can trace back this difference to the constant terms in (A.63) and (A.64).

\section{B Combinatorial identities of partitions}

First, we show the identity $(2.30)$

$$
\begin{aligned}
\sum_{i}(2 i-1) \lambda_{i} & =\sum_{(i, j) \in \lambda}(2 i-1) \\
& =\sum_{j} \sum_{i=1}^{\lambda_{j}^{\prime}}(2 i-1) \\
& =\sum_{j}\left(\lambda_{j}^{\prime}\left(\lambda_{j}^{\prime}+1\right)-\lambda_{j}^{\prime}\right) \\
& =\sum_{j} \lambda_{j}^{\prime 2},
\end{aligned}
$$


where we used that $\sum_{j=1}^{\lambda_{i}} 1=\lambda_{i}$ and $\sum_{i=1}^{\lambda_{j}^{\prime}} i=\frac{\lambda_{j}^{\prime}\left(\lambda_{j}^{\prime}+1\right)}{2}$ above. The second identity to be shown is $(2.34)$

$$
\begin{aligned}
\sum_{i}(2 i-1)^{2} \lambda_{i} & =\sum_{i}\left(4 i^{2}-4 i+1\right) \lambda_{i} \\
& =4 \sum_{i} i^{2} \lambda_{i}-4 \sum_{i} i \lambda_{i}+|\lambda| \\
& =4 \sum_{(i, j) \in \lambda} i^{2}-2 \sum_{j} \lambda_{j}^{\prime 2}-|\lambda| \\
& =4 \sum_{j} \frac{1}{6} \lambda_{j}^{\prime}\left(\lambda_{j}^{\prime}+1\right)\left(2 \lambda_{j}^{\prime}+1\right)-2 \sum_{j} \lambda_{j}^{\prime 2}-|\lambda| \\
& =\frac{4}{3} \sum_{j} \lambda_{j}^{\prime 3}-\frac{1}{3}|\lambda| .
\end{aligned}
$$

We can similarly derive the transformed expression for $\sum_{i}(2 i-1)^{p} \lambda_{i} \propto \sum_{j} \lambda_{j}^{\prime p+1}+\ldots$ for all $p \in \mathbb{N}$. These identities are useful to express parts of the $\mathrm{qBO}_{2}$ eigenvalues in terms of conjugate partitions.

The free boson representation is more useful to integrate the partition function, for example

$$
\sum_{i} \lambda_{i}^{k}=\sum_{r} N_{r} r^{k}
$$

where $N_{r}$ is the number of rows of the partition $\lambda$ with $r$ boxes. In this representation, partitions are denoted by $\lambda=\left(1^{N_{1}} 2^{N_{2}} \cdots\right)$. The challenge here is to obtain the free boson representation for $\sum_{i}(2 i-1)^{q} \lambda_{i}$. Let us see what happens for $q=1$. Let $n_{i} \equiv N_{\lambda_{i}}$ be the number of rows with $\lambda_{i}$ number of boxes, in such a way that

$$
\begin{aligned}
\sum_{i}(2 i-1) \lambda_{i} & =\sum_{i=1}^{n_{1}}(2 i-1) \lambda_{1}+\sum_{i=n_{1}+1}^{n_{1}+n_{2}}(2 i-1) \lambda_{2}+\sum_{i=n_{1}+n_{2}+1}^{n_{1}+n_{2}+n_{3}}(2 i-1) \lambda_{3}+\cdots \\
& =\sum_{k} c_{k} \lambda_{k}
\end{aligned}
$$

where the $\lambda_{k}$ degeneracy is given by

$$
c_{k}=\sum_{i=\sum_{j=1}^{k-1} n_{j}+1}^{\sum_{j=1}^{k} n_{j}}(2 i-1)
$$

Now, let

$$
\tilde{n}_{k}=\sum_{j=1}^{k} n_{j}
$$


then

$$
\begin{aligned}
\sum_{i=\tilde{n}_{k-1}+1}^{\tilde{n}_{k-1}+n_{k}} i & =\sum_{i=1}^{\tilde{n}_{k-1}+n_{k}} i-\sum_{i=1}^{\tilde{n}_{k-1}} i \\
& =\frac{\left(\tilde{n}_{k-1}+n_{k}\right)\left(\tilde{n}_{k-1}+n_{k}+1\right)}{2}-\frac{\tilde{n}_{k-1}\left(\tilde{n}_{k-1}+1\right)}{2} \\
& =\frac{1}{2}\left(n_{k}^{2}+2 \tilde{n}_{k-1} n_{k}+n_{k}\right) \\
& =\frac{1}{2} n_{k}\left(n_{k}+1\right)+\tilde{n}_{k-1} n_{k}
\end{aligned}
$$

and thus

$$
c_{k}=n_{k}\left(n_{k}+1\right)+2 \tilde{n}_{k-1} n_{k}-n_{k}=n_{k}^{2}+2 \tilde{n}_{k-1} n_{k} .
$$

Therefore

$$
\sum_{k} c_{k} \lambda_{k}=\sum_{k}\left(n_{k}^{2}+2 \tilde{n}_{k-1} n_{k}\right) \lambda_{k}
$$

First, using the definition of $n_{k}=N_{\lambda_{k}}$, we notice that

$$
\sum_{k} n_{k}^{2} \lambda_{k}=\sum_{k} N_{\lambda_{k}}^{2} \lambda_{k}=\sum_{r} N_{r}^{2} r
$$

Second, noticing that $\tilde{n}_{0}=0$,

$$
\begin{aligned}
\sum_{k} n_{k} \tilde{n}_{k-1} \lambda_{k} & =\sum_{k} N_{\lambda_{k}} \lambda_{k} \sum_{j=1}^{k-1} N_{\lambda_{j}} \\
& =N_{\lambda_{2}} \lambda_{2} N_{\lambda_{1}}+N_{\lambda_{3}} \lambda_{3}\left(N_{\lambda_{1}}+N_{\lambda_{2}}\right)+N_{\lambda_{4}} \lambda_{4}\left(N_{\lambda_{1}}+N_{\lambda_{2}}+N_{\lambda_{3}}\right)+\ldots \\
& =N_{\lambda_{1}}\left(N_{\lambda_{2}} \lambda_{2}+N_{\lambda_{3}} \lambda_{3}+\ldots\right)+N_{\lambda_{2}}\left(N_{\lambda_{3}} \lambda_{3}+N_{\lambda_{4}} \lambda_{4}+\ldots\right)+N_{\lambda_{3}}\left(N_{\lambda_{4}} \lambda_{4}+\ldots\right) . \\
& =\sum_{i} N_{\lambda_{i}} \sum_{j>i} N_{\lambda_{j}} \lambda_{j} \\
& =\sum_{r} N_{r} \sum_{s=1}^{r-1} N_{s} s .
\end{aligned}
$$

Finally, using all the results above, we get

$$
\sum_{i}(2 i-1) \lambda_{i}=\sum_{r}\left(N_{r}^{2} r+N_{r} \sum_{s=1}^{r-1} N_{s} s\right)=\sum_{r} N_{r} \sum_{s=1}^{r} N_{s} s .
$$

Notice that this can be rewritten as

$$
\sum_{r} N_{r} \sum_{s=1}^{r} N_{s} s=\sum_{r, s} N_{r} A_{r s} N_{s}
$$


where $A_{r s}$ is the (infinite) lower triangular matrix

$$
A=\left(\begin{array}{ccccc}
1 & 0 & 0 & 0 & \ldots \\
1 & 2 & 0 & 0 & \ldots \\
1 & 2 & 3 & 0 & \ldots \\
\ldots & \ldots & \ldots
\end{array}\right) .
$$

We can generalize this derivation to terms like $\sum_{i}(2 i-1) \lambda_{i}^{q}, q>1$ giving

$$
\sum_{r, s} A_{r, s}^{(q)} N_{r} N_{s}, \quad A^{(q)}=\left(\begin{array}{ccccc}
1 & 0 & 0 & 0 & \ldots \\
1 & 2^{q} & 0 & 0 & \ldots \\
1 & 2^{q} & 3^{q} & 0 & \ldots \\
\ldots & \ldots & \ldots
\end{array}\right) .
$$

This derivation also suggests that the terms $\sum_{i}(2 i-1)^{p} \lambda_{i}, p>1$ will be higher-order polynomials in $N_{r}$

$$
A_{r_{1} r_{2} \ldots r_{p+1}}^{(p, 1)} N_{r_{1}} N_{r_{2}} \cdots N_{r_{p+1}}, \quad r_{1} \geq r_{2} \geq \cdots \geq r_{p+1}>0 .
$$

This complicates the integration of these terms in the partition function, which might be related to the theory of integral discriminants [90]. Furthermore, generic terms like $\sum_{i}(2 i-1)^{p} \lambda_{i}^{q}, p, q>1$ will be just like (B.11), but the tensors $A^{(p, q)}$ will have components which are $q$-th powers of integers.

Open Access. This article is distributed under the terms of the Creative Commons Attribution License (CC-BY 4.0), which permits any use, distribution and reproduction in any medium, provided the original author(s) and source are credited.

\section{References}

[1] J.M. Deutsch, Quantum Statistical Mechanics in a Closed System, Phys. Rev. A 43 (1991) 2046.

[2] M. Srednicki, Chaos and Quantum Thermalization, Phys. Rev. E 50 (1994) 888.

[3] J.M. Deutsch, Eigenstate Thermalization Hypothesis, Rept. Prog. Phys. 81 (2018) 082001.

[4] A. Belin, J. De Boer, P. Nayak and J. Sonner, Charged Eigenstate Thermalization, Euclidean Wormholes and Global Symmetries in Quantum Gravity, arXiv:2012.07875 [INSPIRE].

[5] J. de Boer and D. Engelhardt, Remarks on thermalization in 2D CFT, Phys. Rev. D 94 (2016) 126019 [arXiv: 1604.05327] [INSPIRE].

[6] N. Lashkari, A. Dymarsky and H. Liu, Eigenstate Thermalization Hypothesis in Conformal Field Theory, J. Stat. Mech. 1803 (2018) 033101 [arXiv:1610.00302] [INSPIRE].

[7] P. Basu, D. Das, S. Datta and S. Pal, Thermality of eigenstates in conformal field theories, Phys. Rev. E 96 (2017) 022149 [arXiv: 1705.03001] [INSPIRE].

[8] N. Lashkari, A. Dymarsky and H. Liu, Universality of Quantum Information in Chaotic CFTs, JHEP 03 (2018) 070 [arXiv:1710.10458] [INSPIRE]. 
[9] D.A. Roberts and D. Stanford, Two-dimensional conformal field theory and the butterfly effect, Phys. Rev. Lett. 115 (2015) 131603 [arXiv: 1412.5123] [InSPIRE].

[10] J. Maldacena, S.H. Shenker and D. Stanford, A bound on chaos, JHEP 08 (2016) 106 [arXiv: 1503.01409] [INSPIRE].

[11] G. Turiaci and H. Verlinde, On CFT and Quantum Chaos, JHEP 12 (2016) 110 [arXiv: 1603.03020] [INSPIRE].

[12] S. Collier, A. Maloney, H. Maxfield and I. Tsiares, Universal dynamics of heavy operators in $C F T_{2}$, JHEP 07 (2020) 074 [arXiv: 1912.00222] [INSPIRE].

[13] D. Das, Y. Kusuki and S. Pal, Universality in asymptotic bounds and its saturation in $2 D$ CFT, JHEP 04 (2021) 288 [arXiv:2011.02482] [INSPIRE].

[14] J.R. Garrison and T. Grover, Does a single eigenstate encode the full Hamiltonian?, Phys. Rev. X 8 (2018) 021026 [arXiv: 1503.00729] [INSPIRE].

[15] V.V. Bazhanov, S.L. Lukyanov and A.B. Zamolodchikov, Integrable structure of conformal field theory, quantum KdV theory and thermodynamic Bethe ansatz, Commun. Math. Phys. 177 (1996) 381 [hep-th/9412229] [INSPIRE].

[16] V.V. Bazhanov, S.L. Lukyanov and A.B. Zamolodchikov, Integrable structure of conformal field theory. 2. Q operator and DDV equation, Commun. Math. Phys. 190 (1997) 247 [hep-th/9604044] [INSPIRE].

[17] V.V. Bazhanov, S.L. Lukyanov and A.B. Zamolodchikov, Integrable structure of conformal field theory. 3. The Yang-Baxter relation, Commun. Math. Phys. 200 (1999) 297 [hep-th/9805008] [INSPIRE].

[18] J. Cardy, Quantum Quenches to a Critical Point in One Dimension: some further results, J. Stat. Mech. 1602 (2016) 023103 [arXiv: 1507.07266] [INSPIRE].

[19] M. Rigol, V. Dunjko, V. Yurovsky and M. Olshanii, Relaxation in a Completely Integrable Many-Body Quantum System: an Ab Initio Study of the Dynamics of the Highly Excited States of 1d Lattice Hard-Core Bosons, Phys. Rev. Lett. 98 (2007) 050405.

[20] M. Rigol, V. Dunjko and M. Olshanii, Thermalization and Its Mechanism for Generic Isolated Quantum Systems, Nature 452 (2008) 854.

[21] A.C. Cassidy, C.W. Clark and M. Rigol, Generalized Thermalization in an Integrable Lattice System, Phys. Rev. Lett. 106 (2011) 140405.

[22] L. Vidmar and M. Rigol, Generalized Gibbs Ensemble in Integrable Lattice Models, J. Stat. Mech. 2016 (2016) 064007.

[23] L. D'Alessio, Y. Kafri, A. Polkovnikov and M. Rigol, From quantum chaos and eigenstate thermalization to statistical mechanics and thermodynamics, Adv. Phys. 65 (2016) 239 [arXiv: 1509.06411] [INSPIRE].

[24] A. Dymarsky and K. Pavlenko, Generalized Gibbs Ensemble of $2 d$ CFTs at large central charge in the thermodynamic limit, JHEP 01 (2019) 098 [arXiv:1810.11025] [INSPIRE].

[25] A. Maloney, G.S. Ng, S.F. Ross and I. Tsiares, Generalized Gibbs Ensemble and the Statistics of KdV Charges in 2D CFT, JHEP 03 (2019) 075 [arXiv:1810.11054] [InSPIRE].

[26] A. Maloney, G.S. Ng, S.F. Ross and I. Tsiares, Thermal Correlation Functions of KdV Charges in 2D CFT, JHEP 02 (2019) 044 [arXiv:1810.11053] [INSPIRE]. 
[27] A. Dymarsky and K. Pavlenko, Exact generalized partition function of 2D CFTs at large central charge, JHEP 05 (2019) 077 [arXiv: 1812.05108] [INSPIRE].

[28] E.M. Brehm and D. Das, Korteweg-de Vries characters in large central charge CFTs, Phys. Rev. D 101 (2020) 086025 [arXiv: 1901.10354] [INSPIRE].

[29] A. Dymarsky and K. Pavlenko, Generalized Eigenstate Thermalization Hypothesis in $2 D$ Conformal Field Theories, Phys. Rev. Lett. 123 (2019) 111602 [arXiv:1903.03559] [INSPIRE].

[30] S. Datta, P. Kraus and B. Michel, Typicality and thermality in 2d CFT, JHEP 07 (2019) 143 [arXiv: 1904.00668] [INSPIRE].

[31] M. Beşken, S. Datta and P. Kraus, Quantum thermalization and Virasoro symmetry, J. Stat. Mech. 2006 (2020) 063104 [arXiv: 1907.06661] [INSPIRE].

[32] A.G. Abanov, E. Bettelheim and P. Wiegmann, Integrable hydrodynamics of Calogero-Sutherland model: Bidirectional Benjamin-Ono equation, J. Phys. A 42 (2009) 135201 [arXiv: 0810 .5327] [INSPIRE].

[33] V.A. Alba, V.A. Fateev, A.V. Litvinov and G.M. Tarnopolskiy, On combinatorial expansion of the conformal blocks arising from AGT conjecture, Lett. Math. Phys. 98 (2011) 33 [arXiv: 1012.1312] [INSPIRE].

[34] L.F. Alday, D. Gaiotto and Y. Tachikawa, Liouville Correlation Functions from Four-dimensional Gauge Theories, Lett. Math. Phys. 91 (2010) 167 [arXiv:0906.3219] [INSPIRE].

[35] N.A. Nekrasov, Seiberg-Witten prepotential from instanton counting, Adv. Theor. Math. Phys. 7 (2003) 831 [hep-th/0206161] [INSPIRE].

[36] A.V. Litvinov, On spectrum of ILW hierarchy in conformal field theory, JHEP 11 (2013) 155 [arXiv: 1307.8094] [INSPIRE].

[37] B. Feigin, M. Jimbo and E. Mukhin, Integrals of motion from quantum toroidal algebras, J. Phys. A 50 (2017) 464001 [arXiv:1705.07984] [InSPIRE].

[38] A. Litvinov and I. Vilkoviskiy, Liouville reflection operator, affine Yangian and Bethe ansatz, JHEP 12 (2020) 100 [arXiv:2007.00535] [INSPIRE].

[39] A. Dymarsky, K. Pavlenko and D. Solovyev, Zero modes of local operators in $2 d$ CFT on a cylinder, JHEP 07 (2020) 172 [arXiv:1912.13444] [INSPIRE].

[40] S. Detournay, T. Hartman and D.M. Hofman, Warped Conformal Field Theory, Phys. Rev. D 86 (2012) 124018 [arXiv:1210.0539] [INSPIRE].

[41] A. Pérez, D. Tempo and R. Troncoso, Boundary conditions for General Relativity on AdS 3 and the KdV hierarchy, JHEP 06 (2016) 103 [arXiv: 1605.04490] [INSPIRE].

[42] O. Fuentealba et al., Integrable systems with $\mathrm{BMS}_{3}$ Poisson structure and the dynamics of locally flat spacetimes, JHEP 01 (2018) 148 [arXiv:1711.02646] [INSPIRE].

[43] D. Melnikov, F. Novaes, A. Pérez and R. Troncoso, Lifshitz Scaling, Microstate Counting from Number Theory and Black Hole Entropy, JHEP 06 (2019) 054 [arXiv:1808.04034] [INSPIRE].

[44] E. Ojeda and A. Pérez, Boundary conditions for General Relativity in three-dimensional spacetimes, integrable systems and the KdV/mKdV hierarchies, JHEP 08 (2019) 079 [arXiv: 1906.11226] [INSPIRE]. 
[45] A. Dymarsky and S. Sugishita, KdV-charged black holes, JHEP 05 (2020) 041 [arXiv: 2002.08368] [INSPIRE].

[46] A.G. Abanov and P.B. Wiegmann, Quantum hydrodynamics, quantum Benjamin-Ono equation, and Calogero model, Phys. Rev. Lett. 95 (2005) 076402 [cond-mat/0504041] [INSPIRE].

[47] E. Bettelheim, A.G. Abanov and P. Wiegmann, Quantum Shock Waves: The case for non-linear effects in dynamics of electronic liquids, Phys. Rev. Lett. 97 (2006) 246401 [cond-mat/0606778] [INSPIRE].

[48] E. Bettelheim, A.G. Abanov and P. Wiegmann, Nonlinear Dynamics of Quantum Systems and Soliton Theory, J. Phys. A 40 (2007) F193 [nlin/0605006].

[49] P. Wiegmann, Non-Linear hydrodynamics and Fractionally Quantized Solitons at Fractional Quantum Hall Edge, Phys. Rev. Lett. 108 (2012) 206810 [arXiv:1112.0810] [INSPIRE].

[50] T.B. Benjamin, Internal Waves of Permanent Form in Fluids of Great Depth, J. Fluid Mech. 29 (1967) 559.

[51] H. Ono, Algebraic Solitary Waves in Stratified Fluids, J. Phys. Soc. Jap. 39 (1975) 1082.

[52] R. Joseph and R. Egri, Multi-Soliton Solutions in a Finite Depth Fluid, J. Phys. A 11 (1978) L97.

[53] H.H. Chen, Internal-Wave Solitons of Fluids with Finite Depth, Phys. Rev. Lett. 43 (1979) 264.

[54] D.R. Lebedev and A.O. Radul, Generalized internal long waves equations: construction, Hamiltonian structure and conservation laws, Commun. Math. Phys. 91 (1983) 543 [INSPIRE].

[55] G. Bonelli, A. Sciarappa, A. Tanzini and P. Vasko, Six-dimensional supersymmetric gauge theories, quantum cohomology of instanton moduli spaces and $g l(N)$ Quantum Intermediate Long Wave Hydrodynamics, JHEP 07 (2014) 141 [arXiv:1403.6454] [INSPIRE].

[56] P. Koroteev and A. Sciarappa, Quantum Hydrodynamics from Large-N Supersymmetric Gauge Theories, Lett. Math. Phys. 108 (2018) 45 [arXiv:1510.00972] [InSPIRE].

[57] P. Koroteev and A. Sciarappa, On Elliptic Algebras and Large-N Supersymmetric Gauge Theories, J. Math. Phys. 57 (2016) 112302 [arXiv:1601.08238] [INSPIRE].

[58] A. Gorsky, O. Koroteeva, P. Koroteev and A. Vainshtein, On dimensional transmutation in $1+1 D$ quantum hydrodynamics, J. Math. Phys. 61 (2020) 082302 [arXiv:1910.02606] [INSPIRE].

[59] S. Ribault, Conformal field theory on the plane, arXiv:1406.4290 [INSPIRE].

[60] A.A. Belavin, A.M. Polyakov and A.B. Zamolodchikov, Infinite Conformal Symmetry in Two-Dimensional Quantum Field Theory, Nucl. Phys. B 241 (1984) 333 [inSPIRE].

[61] P. Di Francesco, P. Mathieu and D. Senechal, Conformal Field Theory, Graduate Texts in Contemporary Physics, Springer-Verlag, New York (1997) [DOI] [INSPIRE].

[62] A. Castro, D.M. Hofman and G. Sárosi, Warped Weyl fermion partition functions, JHEP 11 (2015) 129 [arXiv: 1508.06302] [INSPIRE].

[63] L. Apolo and W. Song, Bootstrapping holographic warped CFTs or: how I learned to stop worrying and tolerate negative norms, JHEP 07 (2018) 112 [arXiv:1804.10525] [INSPIRE]. 
[64] R. Sakamoto, J. Shiraishi, D. Arnaudon, L. Frappat and É. Ragoucy, Correspondence between conformal field theory and Calogero-Sutherland model, Nucl. Phys. B 704 (2005) 490 [hep-th/0407267] [INSPIRE].

[65] B. Estienne, V. Pasquier, R. Santachiara and D. Serban, Conformal blocks in Virasoro and W theories: Duality and the Calogero-Sutherland model, Nucl. Phys. B 860 (2012) 377 [arXiv:1110.1101] [INSPIRE].

[66] P. Kraus, Lectures on black holes and the $A d S_{3} / C F T_{2}$ correspondence, Lect. Notes Phys. 755 (2008) 193 [hep-th/0609074] [inSPIRE].

[67] A. Maloney and E. Witten, Quantum Gravity Partition Functions in Three Dimensions, JHEP 02 (2010) 029 [arXiv:0712.0155] [INSPIRE].

[68] C.A. Keller and A. Maloney, Poincaré Series, 3D Gravity and CFT Spectroscopy, JHEP 02 (2015) 080 [arXiv: 1407.6008] [INSPIRE].

[69] J.L. Cardy, Operator Content of Two-Dimensional Conformally Invariant Theories, Nucl. Phys. B 270 (1986) 186 [INSPIRE].

[70] S. Pal and Z. Sun, High Energy Modular Bootstrap, Global Symmetries and Defects, JHEP 08 (2020) 064 [arXiv: 2004.12557] [inSPIRE].

[71] K. Huang and S. Weinberg, Ultimate temperature and the early universe, Phys. Rev. Lett. 25 (1970) 895 [INSPIRE].

[72] S. Carlip, Logarithmic corrections to black hole entropy from the Cardy formula, Class. Quant. Grav. 17 (2000) 4175 [gr-qc/0005017] [INSPIRE].

[73] E. Dyer, A.L. Fitzpatrick and Y. Xin, Constraints on Flavored $2 d$ CFT Partition Functions, JHEP 02 (2018) 148 [arXiv:1709.01533] [INSPIRE].

[74] B. Mukhametzhanov and A. Zhiboedov, Modular invariance, tauberian theorems and microcanonical entropy, JHEP 10 (2019) 261 [arXiv: 1904.06359] [INSPIRE].

[75] S. Pal and Z. Sun, Tauberian-Cardy formula with spin, JHEP 01 (2020) 135 [arXiv: 1910.07727] [INSPIRE].

[76] G.E. Andrews and B.C. Berndt, Ramanujan's lost notebook (Part II), Springer New York (2009).

[77] K. Bringmann and A. Milas, W-Algebras, False Theta Functions and Quantum Modular Forms, I, Int. Math. Res. Not. 2015 (2015) 11351.

[78] K. Bringmann, A. Folsom and A. Milas, Asymptotic behavior of partial and false theta functions arising from Jacobi forms and regularized characters, J. Math. Phys. 58 (2017) 011702 [arXiv: 1604.01977] [INSPIRE].

[79] K. Bringmann and C. Nazaroglu, A Framework for Modular Properties of False Theta Functions, arXiv: 1904.05377 [INSPIRE].

[80] D. Das, S. Datta and S. Pal, Charged structure constants from modularity, JHEP 11 (2017) 183 [arXiv: 1706.04612] [INSPIRE].

[81] P. Calabrese and J. Cardy, Quantum Quenches in Extended Systems, J. Stat. Mech. 0706 (2007) P06008 [arXiv:0704.1880] [INSPIRE].

[82] G.A. Kotousov and S.L. Lukyanov, Bethe state norms for the Heisenberg spin chain in the scaling limit, Nucl. Phys. B 947 (2019) 114748 [arXiv: 1906. 07081] [INSPIRE]. 
[83] G.A. Kotousov and S.L. Lukyanov, Spectrum of the reflection operators in different integrable structures, JHEP 02 (2020) 029 [arXiv: 1910.05947] [INSPIRE].

[84] E. Ilievski, J. De Nardis, B. Wouters, J.-S. Caux, F.H. Essler and T. Prosen, Complete Generalized Gibbs Ensembles in an Interacting Theory, Phys. Rev. Lett. 115 (2015) 157201.

[85] O. Fuentealba, H.A. González, M. Pino and R. Troncoso, The anisotropic chiral boson, JHEP 11 (2019) 123 [arXiv: 1909.02699] [INSPIRE].

[86] D.M. Hofman and B. Rollier, Warped Conformal Field Theory as Lower Spin Gravity, Nucl. Phys. B 897 (2015) 1 [arXiv:1411.0672] [INSPIRE].

[87] T. Azeyanagi, S. Detournay and M. Riegler, Warped Black Holes in Lower-Spin Gravity, Phys. Rev. D 99 (2019) 026013 [arXiv:1801.07263] [InSPIRE].

[88] S.L. Lukyanov, Low energy effective Hamiltonian for the XXZ spin chain, Nucl. Phys. B $\mathbf{5 2 2}$ (1998) 533 [cond-mat/9712314] [INSPIRE].

[89] C. Jordan and K. Jordán, Calculus of Finite Differences, vol. 33, American Mathematical Soc. (1965).

[90] A. Morozov and S. Shakirov, Introduction to Integral Discriminants, JHEP 12 (2009) 002 [arXiv: 0903.2595] [INSPIRE]. 